\title{
Relative permeabilities for strictly hyperbolic models of three-phase flow in porous media
}

\author{
Ruben Juanes \\ Tadeusz W. Patzek \\ Department of Civil and Environmental Engineering \\ University of California at Berkeley \\ 631 Davis Hall, Berkeley, CA 94720 \\ Earth Sciences Division \\ Lawrence Berkeley National Lab \\ 1 Cyclotron Road Mailstop 90-1116, Berkeley, CA 94720 \\ Submitted to Transport in Porous Media \\ September 7, 2002
}

\begin{abstract}
Traditional mathematical models of multiphase flow in porous media use a straightforward extension of Darcy's equation, and the key element of these models is the appropriate formulation of the relative permeability functions. It is well known that for one-dimensional flow of three immiscible incompressible fluids, when capillarity is neglected, most relative permeability models used today give rise to regions in the saturation space with elliptic behavior (the so-called elliptic regions). We believe that this behavior is not physical, but rather the result of an incorrect (or incomplete) mathematical model. In this paper we identify necessary conditions that must be satisfied by the relative permeability functions, so that the system of equations
\end{abstract}


describing three-phase flow is strictly hyperbolic everywhere in the saturation triangle. These conditions seem to be in good agreement with pore-scale physics and experimental data.

KEY WORDS: porous media, three-phase flow, relative permeability, strict hyperbolicity, elliptic regions, Oak experiments

\section{Introduction}

Mathematical modeling of multiphase flow in porous media is, to a large extent, still an open issue [51]. In our opinion, the main difficulty stems from the fact that different processes dominate at different scales: capillary forces dominate at the pore scale, while viscous and/or gravity forces dominate at the field scale. ${ }^{1}$ As a result, development of continuum theories of multiphase flow has proven to be an exceptionally challenging task.

The key ingredients of traditional formulations of multiphase flow are mass conservation equations, and a multiphase form of Darcy's equation [6]. Darcy's equation is an approximate form of the fluid momentum balance in creeping flow through porous media. This postulate is supported for singlephase flow by experimental evidence and by volume averaging as a first-order approximation [25]. On the other hand, the usual multiphase flow extension of Darcy's equation due to Muskat [52] does not emanate from averaging of the microscopic equations of multiphase systems [27]. A number of inconsistencies of the standard formulation have been identified, and alternative approaches have been proposed $[5,22,26]$, which are yet to be fully explored.

However, there is still a great interest in Darcy-like formulations, as they are almost universally used in hydrogeology and petroleum engineering. In this framework, success of the formulation depends heavily on the use of "correct" relative permeabilities. Traditionally, they are taken as functions of current fluid saturations alone. This is a very strong assumption, which

\footnotetext{
${ }^{1}$ The sum of the tiny viscous pressure drops in immiscible flow through each pore will eventually exceed the magnitude of capillary pressure, given enough pores along the flow direction. It is capillarity, however, that sets each individual pore's fluid occupancy and the resulting hydraulic resistances. Gravity, in turn, sets the capillary pressure level in each horizontal plane, but capillarity governs the local flow resistances all the same. Although formally correct, the classical comparison of viscous, gravity and capillary forces at the vastly different length scales is misleading. We shall return to this issue in a later paper.
} 
does not account for: (1) hysteretic effects [43,58], which include the past saturation history into the formulation; (2) nonequilibrium effects [5, 26], which introduce the concept of a relaxation time for pore-scale rearrangement of fluid saturations; and (3) the flow regime, determined by the ratios of viscous, capillary, and gravity forces $[44,45]$, which influences the pore-scale mechanisms of fluid displacement.

In this paper we study one-dimensional horizontal flow through porous media of three immiscible, incompressible fluids. The mathematical formulation makes use of the commonly-used extension of Darcy's equation, and the analysis is restricted to relative permeabilities which are functions of fluid saturations alone. This model leads to a $2 \times 2$ system of saturation equations [9]. It was long believed that, when capillarity is ignored, this system of equations would be strictly hyperbolic for any relative permeability functions. This is far from being the case and, in fact, most relative permeability models used today give rise to systems which are not strictly hyperbolic for the entire range of admissible saturations $[7,19,31,36,65,66]$. Loss of strict hyperbolicity typically occurs at bounded regions of the saturation triangle (the so-called elliptic regions), where the system is elliptic in character. We find this behavior disturbing for many reasons, and are of the opinion that elliptic regions are the artifacts of an incorrect mathematical model. The objective of this paper is to show that it is possible to choose relative permeability functions that preserve strict hyperbolicity of the three-phase flow equations, even if the usual extension of Darcy's equation is employed, and the relative permeabilities are assumed to be functions of the fluid saturations alone.

An outline of the paper is as follows. In Section 2 we present the mathematical model of three-phase flow, and introduce the classification of the governing system of equations. In Section 3 we derive necessary conditions that the relative permeability functions must satisfy for the system of equations to be strictly hyperbolic for all admissible saturation states. We show, in Section 4, that the essential condition that needs to be imposed agrees well with experimental data. In Section 5 we draw the main conclusions, and anticipate ongoing and future research. 


\section{Traditional displacement theory of three- phase flow}

In this section we revisit the traditional mathematical formulation of threephase flow of immiscible incompressible fluids. The governing equations are mass conservation equations, with a particular form of the constitutive equation for the mass flux, namely, the extension of Darcy's equation due to Muskat [52]. As noted below, we do not include gravitational and capillary forces in the analysis.

\subsection{System of governing equations}

The explicit assumptions of the mathematical model of three-phase flow we analyze are the following:

1. One-dimensional flow.

2. Immiscible fluids.

3. Incompressible fluids.

4. Homogeneous rigid porous medium.

5. Multiphase flow extension of Darcy's equation.

6. Negligible gravitational effects.

7. Negligible capillary pressure effects.

A detailed derivation of the governing equations can be found elsewhere $[4$, $9,59]$.

By assumption 2, there is no mass transfer between phases and, therefore, one can identify components with phases. The one-dimensional mass conservation equation for the $\alpha$-phase is, in the absence of source terms:

$$
\partial_{t}\left(m_{\alpha}\right)+\partial_{x}\left(F_{\alpha}\right)=0, \quad 0<x<L, t>0,
$$

where $m_{\alpha}$ is the mass density, $F_{\alpha}$ is the mass flux of the $\alpha$-phase, $\partial_{t}(\cdot), \partial_{x}(\cdot)$, denote partial derivatives with respect to time and space, respectively, and $L$ is the length of the domain. For three-phase flow the system consists of aqueous, vapor and liquid phases, corresponding to water $(w)$, gas $(g)$ and 
oil (o) components, respectively. The mass density of each phase is the mass per unit bulk volume of porous medium:

$$
m_{\alpha}=\rho_{\alpha} S_{\alpha} \phi
$$

where $\rho_{\alpha}$ is the density of the $\alpha$-phase, $S_{\alpha}$ is the saturation of the $\alpha$-phase, and $\phi$ is the porosity. By assumptions 3 and 4 , we take the phase densities and the porosity constant. Using the usual multiphase flow extension of Darcy's equation [52] (assumption 5):

$$
F_{\alpha}=-k \frac{k_{r \alpha}}{\mu_{\alpha}} \rho_{\alpha}\left(\partial_{x} p_{\alpha}+\rho_{\alpha} g \partial_{x} z\right)
$$

where $k$ is the absolute permeability, $k_{r \alpha}$ is the relative permeability, $\mu_{\alpha}$ the dynamic viscosity, and $p_{\alpha}$ the pressure of the $\alpha$-phase. Relative permeabilities are assumed to be functions of phase saturations. The gravitational acceleration has absolute value $g$ and points in the negative direction of the $z$-axis. We define the relative mobility of the $\alpha$-phase as

$$
\lambda_{\alpha}:=\frac{k_{r \alpha}}{\mu_{\alpha}}
$$

Neglecting gravitational and capillarity effects (assumptions 6 and 7) the mass conservation equation for the $\alpha$-phase is:

$$
\partial_{t} S_{\alpha}+\partial_{x}\left(-\frac{1}{\phi} k \lambda_{\alpha} \partial_{x} p\right)=0
$$

where $p$ is the pressure, now common to all phases. Since the fluids fill up the pore volume, their saturations add up to one:

$$
S_{w}+S_{o}+S_{g} \equiv 1
$$

Adding the conservation equations for all phases and using the saturation constraint (6), we get the "pressure equation":

$$
\partial_{x}\left(-\frac{1}{\phi} k \lambda_{T} \partial_{x} p\right)=0
$$

where $\lambda_{T}=\lambda_{w}+\lambda_{o}+\lambda_{g}$ is the total mobility. The pressure equation (7) dictates that the total velocity, defined as

$$
v_{T}:=-\frac{1}{\phi} k \lambda_{T} \partial_{x} p
$$


is at most a function of time. We now define the phase velocity,

$$
v_{\alpha}:=\frac{\lambda_{\alpha}}{\lambda_{T}} v_{T}
$$

and the fractional flow of the $\alpha$-phase,

$$
f_{\alpha}:=\frac{v_{\alpha}}{v_{T}}=\frac{\lambda_{\alpha}}{\lambda_{T}} .
$$

With the definitions above, the three-phase flow system is governed by a $2 \times 2$ system of conservation laws,

$$
\partial_{t}\left(\begin{array}{c}
S_{w} \\
S_{g}
\end{array}\right)+v_{T} \partial_{x}\left(\begin{array}{l}
f_{w} \\
f_{g}
\end{array}\right)=\left(\begin{array}{l}
0 \\
0
\end{array}\right),
$$

and the algebraic constraint $S_{o}=1-S_{w}-S_{g}$. The solution is restricted to lie in the saturation triangle:

$$
\mathcal{T}:=\left\{\left(S_{w}, S_{g}\right): S_{w} \geq 0, \quad S_{g} \geq 0, \quad S_{w}+S_{g} \leq 1\right\} .
$$

The saturation triangle is usually represented as a ternary diagram (Figure 1), in which the pair $\left(S_{w}, S_{g}\right)$ corresponds to the triple $\left(S_{w}, S_{g}, S_{o}\right)$, where $S_{o} \equiv 1-S_{w}-S_{g}$.

Equation (11) can be written in vector notation, by defining the vector of unknowns $\boldsymbol{u}=[u, v]^{t}=\left[S_{w}, S_{g}\right]^{t}$, and the flux vector $\boldsymbol{f}=[f, g]^{t}=\left[f_{w}, f_{g}\right]^{t}$, so that one obtains:

$$
\partial_{t} \boldsymbol{u}+v_{T} \partial_{x} \boldsymbol{f}=\mathbf{0}
$$

It is convenient to write the equation above in dimensionless form. Defining the dimensionless space and time coordinates,

$$
\begin{aligned}
x_{D} & :=\frac{x}{L} \\
t_{D} & :=\frac{1}{L} \int_{0}^{t} v_{T}(\tau) \mathrm{d} \tau,
\end{aligned}
$$

Equation (13) reads:

$$
\frac{\partial \boldsymbol{u}}{\partial t_{D}}+\frac{\partial \boldsymbol{f}}{\partial x_{D}}=\mathbf{0}
$$

We shall abuse notation, and understand that the symbols $x$ and $t$ refer to dimensionless space and time coordinates. Therefore, in what follows, we write the system of equations (16) simply as:

$$
\partial_{t} \boldsymbol{u}+\partial_{x} \boldsymbol{f}=\mathbf{0}
$$




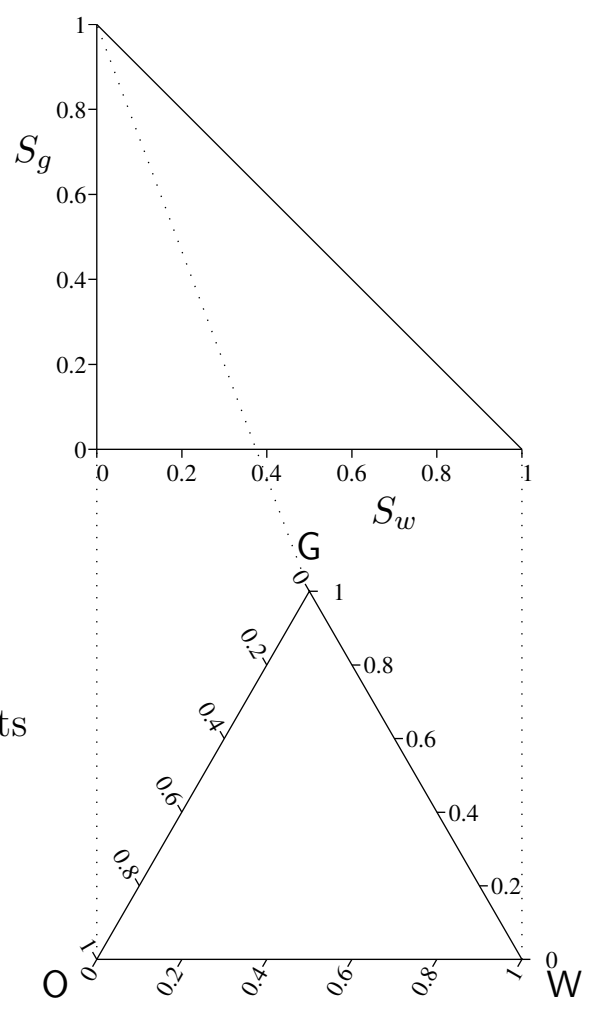

Figure 1. Saturation triangle (top) and ternary diagram (bottom).

\subsection{Flow regions and reduced saturations}

Experimental evidence suggests that there is a threshold saturation for each phase, below which that phase is immobile. This fact has been observed since the earliest two-phase and three-phase flow experiments $[8,46,53,74]$. As a result, three-phase flow takes place only for phase saturations in a region inside the saturation triangle. The nature of these threshold saturations depends on the wettability of the fluids, and on the displacement process [21]. For the most wetting phase, the term "connate" (or "irreducible") saturation would be appropriate both in drainage and imbibition. For the most nonwetting phase, the term "critical" saturation would be applicable in drainage, and "trapped" (or "residual") saturation in imbibition. For the purpose of this paper we lump the terminology above in the term "immobile" saturation, and assume that appropriate values are used for each fluid and for the particular process (saturation path) of interest. 
In principle, these threshold or endpoint saturations do not have to be constant, and the immobile saturation of each phase may vary with the saturations of the other two phases. This is a well-known behavior for the oil phase [41], and several correlations for the "residual" oil saturation have been proposed $[19,20]$. The relative permeability of a phase is zero if that phase is immobile, and it is positive otherwise. For each phase, there will be a line separating the region where the phase is mobile from the region where it is immobile. These lines define regions of one-phase, two-phase and three-phase flow in the ternary diagram.

The three-phase flow region in the space of actual saturations $\boldsymbol{u}=\left(S_{w}, S_{g}\right)^{t}$ can be mapped onto the entire ternary diagram of reduced saturations $\tilde{\boldsymbol{u}}=$ $\left(\tilde{S}_{w}, \tilde{S}_{g}\right)^{t}$, as shown in Figure 2:

$$
\begin{aligned}
\boldsymbol{\varphi}: \mathbb{R}^{2} & \rightarrow \mathbb{R}^{2}, \\
\tilde{\boldsymbol{u}} & \mapsto \boldsymbol{u}=\boldsymbol{\varphi}(\tilde{\boldsymbol{u}}) .
\end{aligned}
$$

We assume that this map is $C^{1}$ invertible and orientation-preserving, so that by simple change of variables we can study three-phase flow in terms of reduced saturations:

$$
\partial_{t}[\boldsymbol{\varphi}(\tilde{\boldsymbol{u}})]+\partial_{x}[\boldsymbol{f}(\boldsymbol{\varphi}(\tilde{\boldsymbol{u}}))]=\mathbf{0} .
$$

Equation (20) can be reduced to its canonical form (see, e.g., [14]) if the regions of mobile and immobile phases are separated by straight lines. In this case the map between actual and reduced saturations is linear,

$$
\boldsymbol{u}=\boldsymbol{\varphi}(\tilde{\boldsymbol{u}})=\boldsymbol{u}_{0}+\varphi^{\prime} \tilde{\boldsymbol{u}}
$$

where $\varphi^{\prime} \equiv \mathrm{D}_{\tilde{\boldsymbol{u}}} \boldsymbol{\varphi}$ is the Jacobian matrix of the mapping, which is constant for a linear map. The system of conservation laws (20) is expressed as

$$
\partial_{t} \tilde{\boldsymbol{u}}+\partial_{x} \tilde{\boldsymbol{f}}=\mathbf{0}
$$

where the newly defined flux $\tilde{\boldsymbol{f}}$ is related to the original flux function as follows:

$$
\tilde{\boldsymbol{f}}(\tilde{\boldsymbol{u}}):=\left(\boldsymbol{\varphi}^{\prime}\right)^{-1} \boldsymbol{f}(\boldsymbol{\varphi}(\tilde{\boldsymbol{u}})) .
$$

Relative permeabilities and, consequently, fractional flows, are expressed in Equation (22) as functions of reduced saturations. By definition, they take 


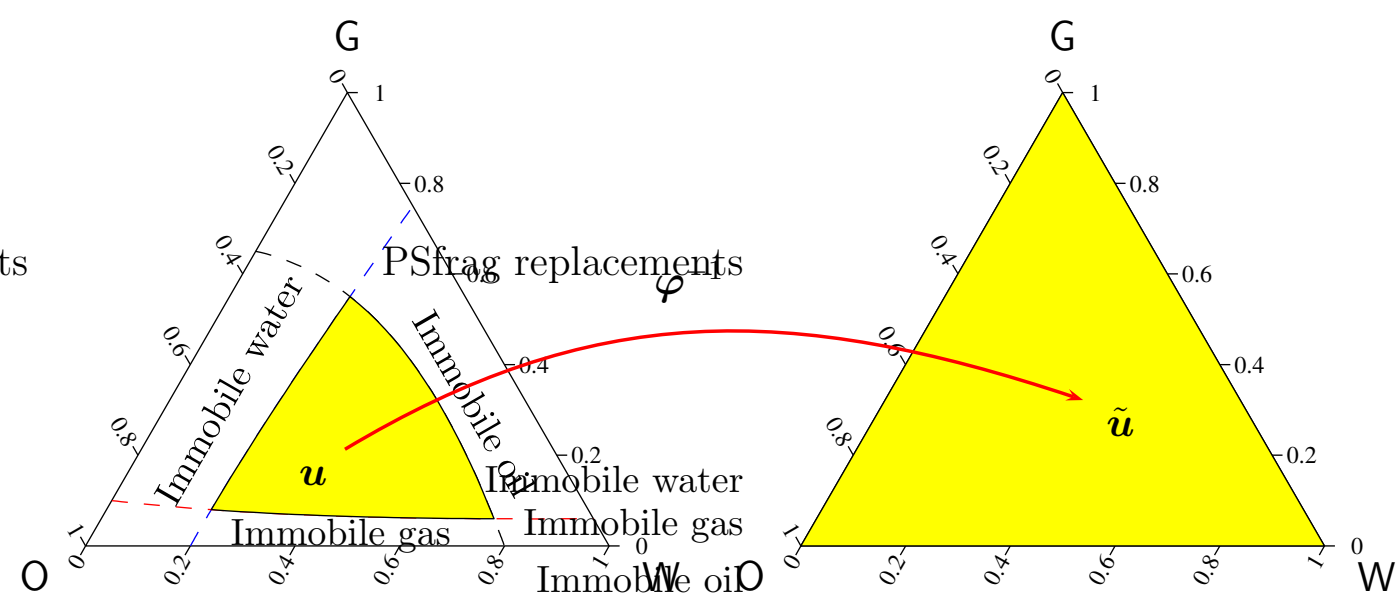

(a) Space of actual saturations

(b) Space of reduced saturations

Figure 2. Schematic of the map between (a) the space of actual saturations, and (b) the space of reduced saturations. The lines defining immobile phases subdivide the ternary diagram into regions of three-phase (center), two-phase (along the edges) and one-phase flow (near the vertices). The three-phase flow region (shaded area) is mapped onto the entire ternary diagram.

a zero value along one of the edges of the ternary diagram, and are positive everywhere else. To simplify notation we shall drop the tildes from Equation (22) and write

$$
\partial_{t} \boldsymbol{u}+\partial_{x} \boldsymbol{f}=\mathbf{0}
$$

but still refer to the system in terms of reduced saturations.

Remark. If the lines defining immobile regions are straight lines parallel to the edges of the ternary diagram, the tree-phase flow region is an equilateral triangle. In this particular case, reduced saturations take the following simple expression:

$$
\tilde{S}_{\alpha}:=\frac{S_{\alpha}-S_{\alpha i}}{1-\left(S_{w i}+S_{o i}+S_{g i}\right)}, \quad \alpha=w, o, g,
$$

where $S_{\alpha i}$ is the immobile saturation (now constant) of the $\alpha$-phase. 


\subsection{Character of the system of equations}

For the purpose of the classification of the system (24), we write it in quasilinear form:

$$
\partial_{t} \boldsymbol{u}+\boldsymbol{A}(\boldsymbol{u}) \partial_{x} \boldsymbol{u}=\mathbf{0}
$$

where

$$
\boldsymbol{A}(\boldsymbol{u}):=\boldsymbol{f}^{\prime}(\boldsymbol{u}) \equiv \mathrm{D}_{\boldsymbol{u}} \boldsymbol{f} \equiv\left(\begin{array}{ll}
f_{, u}(\boldsymbol{u}) & f_{, v}(\boldsymbol{u}) \\
g_{, u}(\boldsymbol{u}) & g_{, v}(\boldsymbol{u})
\end{array}\right)
$$

is the Jacobian matrix of the system at point $\boldsymbol{u}$. Subscripts after a comma denote differentiation $\left(e . g ., f_{, u} \equiv \partial_{u} f\right)$. The classification of the $2 \times 2$ system of first-order partial differential equations (26) is based on the properties of the characteristic curves $[67,75]$.

Definition. A characteristic curve of (26) is a curve on the $(x, t)$-plane, along which $\partial_{t} \boldsymbol{u}$ and $\partial_{x} \boldsymbol{u}$ cannot be specified (if indeed they can be determined at all) if the initial data are prescribed along that same curve.

From the definition above, it follows $[14,75]$ that a characteristic curve of the system (26) associated with a classical solution $\boldsymbol{u}(x, t)$ is a function $x=x(t)$, which is an integral curve of the ordinary differential equation

$$
\frac{\mathrm{d} x}{\mathrm{~d} t}=\nu(\boldsymbol{u}(x, t))
$$

where $\nu(\boldsymbol{u})$ is an eigenvalue of the Jacobian matrix $\boldsymbol{A}(\boldsymbol{u})$. As a result, the classification of the system (26) reduces to analyzing the behavior of the eigenvalue problem

$$
\boldsymbol{A r}=\nu \boldsymbol{r},
$$

where the Jacobian matrix $\boldsymbol{A}$, the eigenvalue $\nu$, and the right eigenvector $\boldsymbol{r}$, are evaluated at a point $\boldsymbol{u}$.

For the eigenvalue problem (29) with a $2 \times 2$ real matrix, it is wellknown [11] that there exists a real nonsingular matrix $\boldsymbol{T}$ such that, after the change of variables $\boldsymbol{z}=\boldsymbol{T} \boldsymbol{r}$, the equivalent eigenvalue problem

$$
\left(T A T^{-1}\right) z=\nu z
$$

has a real coefficient matrix $\boldsymbol{J}:=\boldsymbol{T} \boldsymbol{A} \boldsymbol{T}^{-1}$, which has one of the following canonical forms:

1. $\left(\begin{array}{ll}\lambda & 0 \\ 0 & \mu\end{array}\right), \quad \lambda \neq \mu$ 

2. $\left(\begin{array}{cc}\alpha & -\beta \\ \beta & \alpha\end{array}\right), \quad \beta \neq 0$,
3. $\left(\begin{array}{cc}\lambda & 0 \\ 0 & \lambda\end{array}\right)$,
4. $\left(\begin{array}{ll}\lambda & 1 \\ 0 & \lambda\end{array}\right)$.

These four canonical forms provide the basis for the classification of the system of first-order partial differential equations. Following the terminology in [75], we denote the system whose Jacobian matrix has the canonical form of cases 1 through 4 above, respectively:

1. Strictly hyperbolic. The eigenvalue problem has two real, distinct eigenvalues. The Jacobian matrix is diagonalizable and there are two real and linearly independent eigenvectors [1]. Therefore, the system has two distinct families of characteristic curves, which carry waves traveling at different characteristic speeds.

2. Elliptic. The eigenvalues are complex conjugates, and there are no real characteristic curves that may act as carriers of possible discontinuities in the solution $[14,75]$.

3. Nonstrictly hyperbolic. In this case, there is a double real eigenvalue, and the Jacobian matrix is diagonalizable. Every direction is characteristic, so one can pick any two linearly independent vectors as eigenvectors. The system is hyperbolic (real eigenvalues and linearly independent eigenvectors) but not strictly hyperbolic (which requires that the eigenvalues be distinct).

4. Parabolic. The system has a real, double eigenvalue, and the Jacobian matrix is defective (non-diagonalizable). There is only one eigenvector and, therefore, there is only one real characteristic direction.

This completes the classification of the system at any given state $\boldsymbol{u}$. It is important to note that this classification is restricted to $2 \times 2$ real systems of first-order equations. As we shall see, the character of the system may be different in different regions of the phase space (i.e., the saturation triangle). 
Remark. The eigenvalues $\nu_{p}, p=1,2$ of the original Jacobian matrix (27) are given by

$$
\nu_{1,2}=\frac{1}{2}\left[f_{, u}+g_{, v} \mp \sqrt{\left(f_{, u}-g_{, v}\right)^{2}+4 f_{, v} g_{, u}}\right] .
$$

The physical interpretation of the eigenvalues (when they are real) is the characteristic speeds at which waves describing changes in saturation propagate through the domain. In the strictly hyperbolic case, there exist two distinct waves which travel at different characteristic speeds. It is common to use the terms slow wave and fast wave for the waves associated with the smaller and larger eigenvalue, respectively.

Remark. The right eigenvectors $\boldsymbol{r}_{p}=\left[r_{p u}, r_{p v}\right]^{t}, p=1,2$, which correspond to eigenvalues $\nu_{p}, p=1,2$, respectively, are calculated by the following expressions:

$$
\begin{aligned}
& \frac{r_{1 v}}{r_{1 u}}=\frac{\nu_{1}-f_{, u}}{f_{, v}}=\frac{g_{, u}}{\nu_{1}-g_{, v}}, \\
& \frac{r_{2 u}}{r_{2 v}}=\frac{f_{, v}}{\nu_{2}-f_{, u}}=\frac{\nu_{2}-g_{, v}}{g_{, u}} .
\end{aligned}
$$

When they are real, the right eigenvectors correspond to the directions (in the phase space) of admissible changes in saturation. In the strictly hyperbolic case, the changes in saturation associated with the slow (resp. fast) wave have a direction dictated by $\boldsymbol{r}_{1}\left(\right.$ resp. $\left.\boldsymbol{r}_{2}\right)$ and propagate with velocity $\nu_{1}$ (resp. $\nu_{2}$ ).

\section{Relative permeabilities for strict hyperbol- icity}

In this section we investigate the character of the system of equations (24) describing one-dimensional three-phase flow of immiscible incompressible fluids. The objectives are to better understand the interplay between relative permeabilities and the nature of the system, and to derive conditions on phase mobilities (and ultimately on relative permeabilities) as functions of saturations so as to preserve strict hyperbolicity. 


\subsection{Loss of strict hyperbolicity in traditional models}

It was long believed that the system (24) was strictly hyperbolic for any relative permeability functions. Then, the theory of Lax [42] as extended by Liu [47,48] would apply. However, Bell et al. [7] showed that the system is not necessarily hyperbolic. In particular, they observed that Stone I [68] relative permeabilities gave rise to elliptic regions inside the saturation triangle. Elliptic regions are portions of the saturation triangle where the eigenvalues are complex, so the system is locally elliptic rather than hyperbolic. In subsequent publications $[19,31,36,65,66]$, it was shown that occurrence of elliptic regions is the rule rather than exception for the most common relative permeability models.

Loss of strict hyperbolicity of three-phase flow models was analyzed by Shearer [65] and Holden [31]. They also used reduced saturations, and therefore limited their analysis to the three-phase flow region. From the point of view of studying the character of the system, this assumption is not particularly restrictive, for it can be shown that elliptic regions cannot exist in the one-phase and two-phase flow regions $[18,37]$.

The analysis of Shearer [65] and Holden [31] starts by assuming the behavior of relative permeabilities in two-phase flow. Relative permeabilities (and, therefore, relative mobilities) of both phases, say water and gas, are taken as functions of the reduced water saturation:

$$
\lambda_{w}=\lambda_{w}(u), \quad \lambda_{g}=\lambda_{g}(u)
$$

These functions are assumed to have a zero value and a zero derivative at their endpoint saturations, i.e.,

$$
\lambda_{w}(0)=\lambda_{w}^{\prime}(0)=0, \quad \lambda_{g}(1)=\lambda_{g}^{\prime}(1)=0 .
$$

This behavior, which is taken for granted without further discussion, is then used as a guidance to impose conditions on the edges of the saturation triangle.

In three-phase flow, relative permeabilities are assumed to be functions of the water and gas reduced saturations:

$$
\lambda_{w}=\lambda_{w}(u, v), \quad \lambda_{g}=\lambda_{g}(u, v), \quad \lambda_{o}=\lambda_{o}(u, v) .
$$

Shearer [65] imposes two types of conditions on the edges: 
1. Consistency conditions (termed "(B-L) conditions"), which reduce threephase relative mobilities to the assumed behavior for two-phase flow (Equation (35)), when one of the phases is not mobile. For example, on the edge of zero reduced gas saturation $(v=0,0<u<1)$, it is required:

$$
\begin{aligned}
\lambda_{w}(0,0)=\lambda_{w, u}(0,0) & =0, \\
\lambda_{g}(u, 0) & \equiv 0, \\
\lambda_{o}(1,0)=\lambda_{o, u}(1,0) & =0 .
\end{aligned}
$$

Similar conditions hold on the other two edges.

2. A first interaction condition (termed "(I1) condition"), that limits the effect of the immobile phase on the flow, compared to that of the other two phases. For instance, on the edge $v=0,0<u<1$ :

$$
\lambda_{g, v}<\frac{\lambda_{o} \lambda_{w, u}-\lambda_{w} \lambda_{o, u}}{\lambda_{w}+\lambda_{o}},
$$

and similarly on the other two edges. This condition, together with the consistency conditions above, effectively imposes that the right eigenvector associated with the fast characteristic speed, $\boldsymbol{r}_{2}$, is parallel to the edge. It is important to note that condition (38) is immediately satisfied if $\lambda_{g, v}(u, 0)=0$, that is, if the derivative of the gas mobility along the normal direction to the edge is zero.

An additional condition is imposed near the vertices:

3. A second interaction condition (termed "(I2) condition"), imposes that the right eigenvector associated with the fast characteristic, $\boldsymbol{r}_{2}$, points into the saturation triangle for states $\boldsymbol{u}$ near a vertex. A sufficient (but not necessary) condition that satisfies this requirement is that the off-diagonal terms of the Jacobian matrix $\boldsymbol{A}(\boldsymbol{u})$, are positive. For example, near the vertex of immobile water and gas (near $u=v=0$ ), it is sufficient to have

$$
\begin{aligned}
& \lambda_{w, v}\left(\lambda_{g}+\lambda_{o}\right)-\lambda_{w}\left(\lambda_{g, v}+\lambda_{o, v}\right)>0 \\
& \lambda_{g, u}\left(\lambda_{w}+\lambda_{o}\right)-\lambda_{g}\left(\lambda_{w, u}+\lambda_{o, u}\right)>0 .
\end{aligned}
$$

Holden [31] imposes very similar conditions. The following is required on the edges: 
1. The value of the relative mobility of a phase is zero along the edge of zero reduced saturation of that phase, i.e.,

$$
\begin{array}{ll}
\lambda_{w}=0 & \text { on } u=0,0<v<1, \\
\lambda_{g}=0 & \text { on } v=0,0<u<1, \\
\lambda_{o}=0 & \text { on } v=1-u, 0<u<1 .
\end{array}
$$

2. The derivative of the relative mobility of a phase along the normal direction to the edge of zero reduced saturation is also zero:

$$
\begin{aligned}
\lambda_{w, u}=0 & \text { on } u=0,0<v<1, \\
\lambda_{g, v}=0 & \text { on } v=0,0<u<1, \\
-\lambda_{o, u}-\lambda_{o, v}=0 & \text { on } v=1-u, 0<u<1 .
\end{aligned}
$$

We note that the conditions above on the normal derivatives imply that the "(I1) interaction condition" of Shearer is immediately satisfied and, as a result, the eigenvector of the fast family is parallel to the edges of the saturation triangle.

The properties near the corners are introduced next:

3. Holden [31] considered three possible types of behavior near the vertices of the saturation triangle, based on the sign of the off-diagonal terms of the Jacobian matrix, $f_{, v}, g_{, u}$ :

(A1) Both are positive: $f_{, v}>0, g_{, u}>0$.

(A2) Have different sign: $f_{, v} g_{, u}<0$.

(A3) Both are negative: $f_{, v}<0, g_{, u}<0$.

Using a wettability argument, it is suggested that condition (A1) is the most reasonable in all three corners. This condition implies that the "(I2) interaction condition" of Shearer is automatically satisfied.

We can summarize the conditions imposed by Shearer [65] and Holden [31] as follows (Figure 3):

1. The right eigenvector associated with the fast characteristic family, $\boldsymbol{r}_{2}$, is parallel to the edges of the triangle of reduced saturations. 


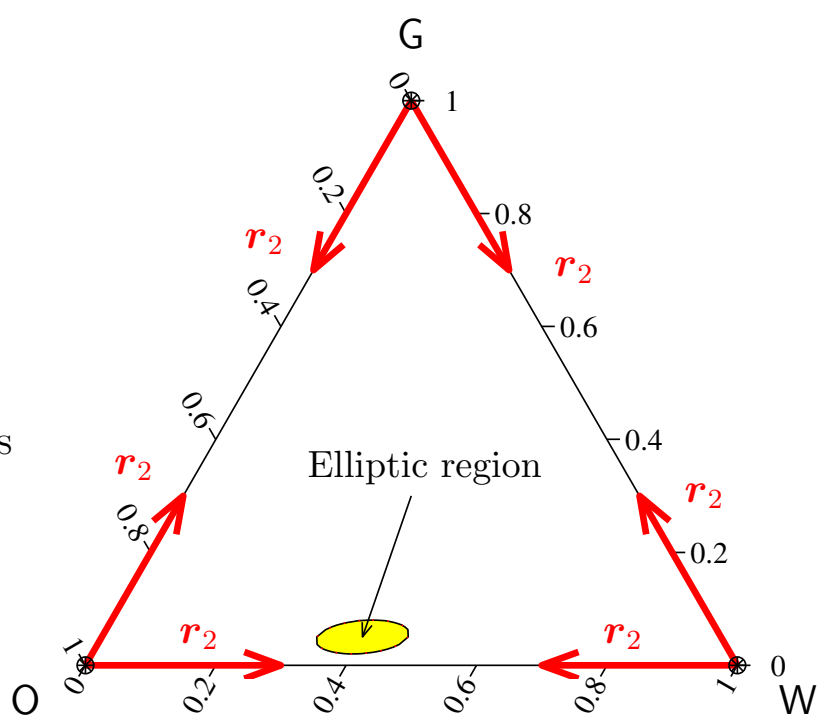

Figure 3. Schematic representation of the direction of fast eigenvectors $\boldsymbol{r}_{2}$ along the edges of the saturation triangle for the models analyzed by Shearer [65] and Holden [31]. For models of this type, vertices are umbilic points, and there must be an elliptic region inside the saturation triangle, usually very close to the oil-water edge.

2. The fast eigenvector $\boldsymbol{r}_{2}$ points into the triangle, for saturation states near the vertices.

The assumed behavior at the edges and corners of the saturation triangle has a profound impact on the character of the system. The first consequence is that each vertex of the saturation triangle is an umbilic point, i.e., eigenvalues are equal and the system is not strictly hyperbolic at those points. The second consequence is that, in general, an elliptic region must exist inside the saturation triangle. This general result can be proved using ideas of projective geometry [31, 63, 65].

Naturally, the question of whether elliptic regions in the saturation space are physically-plausible arises. We defer a full discussion on this topic to a forthcoming publication [39]. However, we briefly point out some of the reasons why local elliptic behavior seems to be an undesirable artifact of the mathematical model, rather than a necessary consequence dictated by physics. 
1. The first remark that one should bear in mind is that Equation (24) is a system of first-order equations in space-time coordinates. Thus, the physical meaning of a system with mixed elliptic/hyperbolic behavior is very different from that when the independent variables are two space coordinates, such as in steady transonic flow [13,40]. In the former case, "initial data" should be imposed in such a way that the principle of causality ${ }^{2}$ is not violated [19].

2. Saturation states inside the elliptic region give rise to linearly ill-posed problems. More precisely, a bounded solution to the linearized Cauchy problem (initial value problem on an unbounded domain) does not exist when arbitrarily close - but not equal - asymptotic left and right states are inside the elliptic region. This fact is in frontal disagreement with the notion of three-phase flow displacement, where one expects a bounded and monotonic transition between the right (initial) state and the left (injected) state. However, the question of whether ill-posedness remains when nonlinear effects are accounted for is still unclear [2,30].

3. In connection with the loss of well-posedness of the problem, appropriate entropy conditions have not yet been found so as to allow both existence and uniqueness of solutions $[2,3]$. Although a few qualitative properties are known [28-30], a complete theory of mixed elliptic/hyperbolic systems does not yet exist.

4. More specifically to models of three-phase flow in porous media, it has been found [36] that different models matching experimental data equally well, produce elliptic regions in opposite corners of the saturation triangle. This result suggests a nonphysical arbitrariness to the location of elliptic behavior in phase space for traditional models of three-phase flow.

5. If capillarity is introduced in the formulation and a traveling wave solution is sought for the Riemann problem (unbounded domain, and piecewise constant initial data with a single discontinuity), the critical points of the associated $2 \times 2$ dynamical system are spiral points [11].

\footnotetext{
2 "The causality of natural processes may be interpreted as implying that the conditions in a body at time $t$ are determined by the past history of the body, and that no aspect of its future behavior need to be known in order to determine all of them." [71]
} 
If a traveling wave solution exists, it will necessarily present a spirallike behavior near the critical points, which translates into oscillatory (nonmonotonic) saturation profiles. Validity of this type of solution is questionable on several counts: (1) as the capillarity effects are taken to zero, oscillations collapse into a singular shock, of dubious physical interpretation; (2) introducing "sufficient" capillarity will not cure the problem of oscillatory behavior, as the spiral-like orbit will persist asymptotically.

6. Numerical simulations seem to corroborate, at least in first instance, the nonphysical behavior of solutions inside the elliptic region [7,34]. For arbitrarily close left and right states inside the elliptic region, the solution develops wildly oscillatory waves, which are never observed in experiments. Moreover, the wave pattern is unstable with respect to the initial states [7].

The only relative permeability models which do not produce elliptic regions (under the assumed behavior at the edges and corners) are those where the relative permeability of a phase depends solely on the saturation of that phase $[49,70]$. This behavior is not supported by experimental results [56] and pore-scale considerations [72]. For models of this type, the elliptic region (where eigenvalues are complex conjugates), shrinks to an isolated umbilic point (a saturation state with a real double eigenvalue), which cannot be removed by further approximation of the relative permeabilities. As shown by Holden [31], it may be possible to reduce the elliptic region to an isolated umbilic point for more general relative permeability models. However, this requires a continuous deformation of the relative permeability functions, which is physically unappealing. Umbilic points also act as "repellers" for classical waves $[23,24,50]$ and, as a result, solutions to the nonstrictly hyperbolic system require nonclassical waves (termed transitional waves [32]). The most salient features of these solutions are that: (1) they are sensitive to the particular form of the diffusion term due to capillary effects [33]; (2) the saturation path may be the same for wildly different initial and injected states $[17,18,23,24]$; and (3) in WAG displacement, the intermediate oil bank may be split in two [50].

From the observations above, it is difficult to justify the physical relevance of mathematical singularities like elliptic regions and umbilic points $[9,10,19$, $51,61,66,70]$. In fact, the presence of elliptic regions in models of three-phase flow has not yet been successfully justified on physical grounds. The first 
attempt in this direction we are aware of is the recent paper by Jackson and Blunt [34], which we review elsewhere $[38,39]$. We are of the opinion that these singularities are mere artifacts of an incorrect mathematical model. Inappropriateness of the formulation may have several sources, which we discuss in more detail in a separate publication [39]. For the purpose of this paper, where the traditional multiphase flow extension of Darcy's equation is used, the element of the formulation that first needs to be revisited is the relative permeability model and, in particular, the assumed behavior at the edges of the saturation triangle. In fact, it is widely recognized that the slope of experimental relative permeabilities near the endpoints is often ill-defined [19].

\subsection{Conditions for strict hyperbolicity}

The generic approach in the existing literature can be summarized as follows: a certain behavior of the relative permeabilities is assumed, and loss of strict hyperbolicity inside the saturation triangle is inferred. We adopt the opposite viewpoint: we assume that the system is strictly hyperbolic, and investigate the conditions on relative permeabilities as functions of saturation such that strict hyperbolicity is preserved. In doing so we keep, however, the restriction that eigenvectors should not rotate along edges of the ternary diagram. What is more, we require that one of the eigenvectors is parallel to any given edge. The reason is to preserve the property that the edges of the ternary diagram are invariant lines for the system of equations [65], i.e., if one phase is immobile everywhere at the initial time, that phase remains immobile (zero reduced saturation) everywhere at all times. This is a very simplistic condition, which in fact does not have to hold if curved lines defining immobile saturations are used, or if hysteretic effects are considered (in this case, the boundary of the three-phase flow region would change with the saturation path of interest).

It is easy to see that the requirement above (one eigenvector parallel to each edge), precludes the possibility of having a strictly hyperbolic system everywhere along the edges of the ternary diagram. There are two different ways in which strict hyperbolicity may fail on the boundary of the saturation triangle:

1. The system is strictly hyperbolic at all three vertices. For vertices to be strictly hyperbolic, eigenvectors lying on each of the two edges must be 


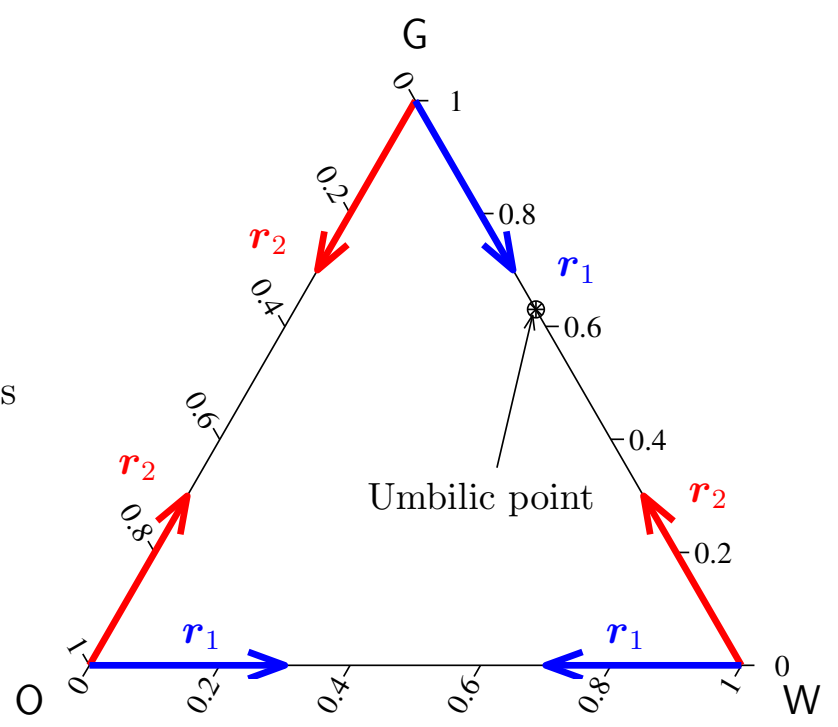

Figure 4. Schematic representation of the direction of fast $\left(\boldsymbol{r}_{2}\right)$ and slow $\left(\boldsymbol{r}_{1}\right)$ eigenvectors along the edges of the saturation triangle models with strictly hyperbolic vertices. An umbilic point must exist somewhere on the WG edge, where eigenvalues of the slow and fast characteristic families coincide.

of different family (e.g., at the $\mathrm{O}$ corner, $\boldsymbol{r}_{1}$ is parallel to the OW edge and $\boldsymbol{r}_{2}$ is parallel to the OG edge). But then, there must exist at least one edge that has a parallel eigenvector of the fast family near one vertex, and of the slow family near the other vertex. Inevitably, an umbilic point -where characteristic speeds of the slow and fast characteristic families coincide - must exist somewhere on this edge, because eigenvectors are not allowed to rotate along the edge (Figure 4).

2. At least one of the vertices is an umbilic point. As we show below, it is possible to have a model that will be nonstrictly hyperbolic at the $\mathrm{G}$ vertex and strictly hyperbolic everywhere else (Figure 5).

Having the considerations above in mind, the key observation is that, whenever gas is present as a continuous phase, the mobility of gas is much higher than that of the other two fluids (water and oil). To honor this physical behavior, we associate fast characteristic paths with displacements involving changes in gas saturation, even in the region of small gas saturation. 


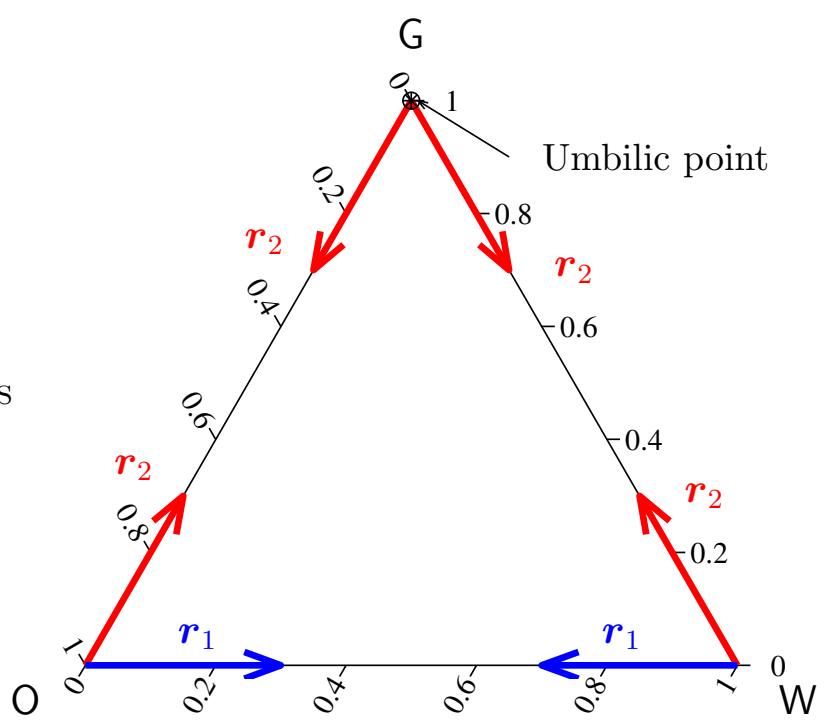

Figure 5. Schematic representation of the direction of fast $\left(\boldsymbol{r}_{2}\right)$ and slow $\left(\boldsymbol{r}_{1}\right)$ eigenvectors along the edges of the saturation triangle for the type of models we propose. The systems is strictly hyperbolic everywhere inside the saturation triangle, and the only umbilic point is located at the $G$ vertex, where the fast paths corresponding to the OG and WG edges coalesce.

The immediate consequence is that the eigenvector associated with the fast family of characteristics $\left(\boldsymbol{r}_{2}\right)$ is transversal — and not parallel - to the oilwater edge of the ternary diagram (Figure 5). As we shall see, this conceptual picture permits that the system will be strictly hyperbolic everywhere inside the saturation triangle. The $\mathrm{G}$ vertex, corresponding to $100 \%$ reduced gas saturation, remains an umbilic point because fast paths corresponding to the OG and WG edges coalesce. This umbilic point could be further removed if one allows for fast eigenvectors to rotate along the OG and WG edges. This was not done here to prevent saturation paths from falling outside the three-phase flow region.

Let us recapitulate the conceptual picture expressed in Figure 5:

1. Along the oil-water (OW) edge, the eigenvector associated with the slow characteristic family $\left(\boldsymbol{r}_{1}\right)$ is parallel to the edge. The system is strictly hyperbolic everywhere along the edge, including the $\mathrm{O}$ and $\mathrm{W}$ vertices. 
2. Along the oil-gas ( $\mathrm{OG}$ ) and water-gas (WG) edges, the eigenvector associated with the fast characteristic family $\left(\boldsymbol{r}_{2}\right)$ is parallel to these edges. The system is strictly hyperbolic everywhere along the edges except at the $G$ vertex, which is an umbilic point.

Below we present a systematic study of the general conditions that ensure strict hyperbolicity of the system. On each edge we identify two types of conditions. Condition I enforces that eigenvectors of the appropriate family are parallel to the edge. Condition II guarantees strict hyperbolicity of the system along the edge. The latter condition is further specialized to both vertices of each edge, which provides additional insight into the behavior of the relative permeabilities. The analytical developments are expressed most effectively in terms of water and gas fractional flows ( $f$ and $g$, respectively) and their derivatives with respect to water and gas saturations $(u$ and $v$, respectively). We then translate these requirements into conditions that the relative permeabilities must satisfy. We emphasize that relative permeabilities and, therefore, relative mobilities, are assumed to be functions of saturations only:

$$
\lambda_{w}=\lambda_{w}(u, v), \quad \lambda_{g}=\lambda_{g}(u, v), \quad \lambda_{o}=\lambda_{o}(u, v) .
$$

\subsubsection{Analysis along the OW edge.}

This edge corresponds to the line of zero reduced gas saturation, $v=0$. The mathematical condition for the slow eigenvector to be parallel to the OW edge $\left(\boldsymbol{r}_{1}=[1,0]^{t}\right)$ is:

$$
g_{, u}=0
$$

When expressed in terms of mobilities, Condition I above reads:

$$
\lambda_{g, u}=0
$$

that is, the derivative of the gas relative mobility with respect to water saturation is zero. This condition is immediately satisfied for any model, as the gas mobility is identically zero along this edge.

For the eigenvector $\boldsymbol{r}_{1}$ to be parallel to the edge, it is also necessary that the denominator in Equation (32) is different from zero:

$$
\nu_{1}-g_{, v} \neq 0 \text {. }
$$


Moreover, introducing Equation (43) into the expression of the eigenvalues (Equation (31)), one obtains:

$$
\nu_{1,2}=\left\{\begin{array}{l}
f_{, u} \\
g_{, v}
\end{array}\right.
$$

Condition (45), together with the condition for strict hyperbolicity, $\nu_{1}<\nu_{2}$, implies that

$$
H_{o w}:=g_{, v}-f_{, u}>0
$$

which is equivalent to

$$
\lambda_{g, v}>\lambda_{w, u}-\lambda_{T, u} \frac{\lambda_{w}}{\lambda_{T}}
$$

Condition II above is the fundamental requirement for strict hyperbolicity of the system of equations of three-phase flow. When this condition is evaluated at the vertices of the OW edge, one obtains:

$$
\begin{array}{ll}
\lambda_{g, v}>\lambda_{w, u} & \text { at the } \mathrm{O} \text { vertex, } \\
\lambda_{g, v}>-\lambda_{o, u} & \text { at the } \mathrm{W} \text { vertex, }
\end{array}
$$

where the inequalities above are strict. In particular, Equations (49)-(50) require that the gas relative permeability does not have zero-derivative at its endpoint saturation. A summary of the conditions at the OW edge is given in Table 1. We make the following important remarks:

1. The requirement of a nonzero endpoint slope of the gas relative permeability is a necessary condition for strict hyperbolicity, which is violated by the models of all previous studies on this subject.

2. This behavior of gas relative permeability is in good agreement with experimental observations of two-phase $[8,21,53,57,60,74]$ and threephase flow $[46,54-56,62,64]$, both in drainage and imbibition. We demonstrate this agreement in Section 4.

3. A finite positive slope for the gas relative permeability can also be justified from the point of view of pore-scale processes. Gas is always the nonwetting fluid, so gas flow takes place through the middle region of the pores (bulk flow). In a drainage process, gas flow starts with the first percolating cluster. Likewise, in an imbibition process, gas 
Table 1. Summary of conditions along the OW edge

\begin{tabular}{crrll} 
Condition & Frac. flows & & Mobilities \\
\hline I & $g_{, u}=0$ & $\Leftrightarrow$ & $\lambda_{g, u}=0$ \\
II & $g_{, v}-f_{, u}>0$ & $\Leftrightarrow$ & $\lambda_{g, v}>\lambda_{w, u}-\lambda_{T, u} \frac{\lambda_{w}}{\lambda_{T}}$ \\
II at O & & & $\lambda_{g, v}>\lambda_{w, u}$ \\
II at W & & & $\lambda_{g, v}>-\lambda_{o, u}$ \\
\hline
\end{tabular}

flow ceases with the last trapped cluster. In both cases, the transition between zero flow and nonzero flow is rather abrupt, thus justifying the existence of a positive slope at the endpoint of the relative permeability curve.

4. In contrast, near their endpoint saturations, the most wetting and intermediate wetting fluids flow through a continuous network of films [15] (corner flow and/or film flow). The effective cross-sectional area of this network varies depending on the local level of capillary pressure. Since the fluid conductance is proportional to the cross-sectional area, it seems plausible that that the relative permeability will approach zero as a quadratic or higher-order power function of saturation and, thus, with zero slope.

\subsubsection{Analysis along the OG edge.}

This edge corresponds to the line of zero reduced water saturation, $u=0$. A necessary condition for the fast eigenvector to be parallel to the OG edge $\left(\boldsymbol{r}_{2}=[0,1]^{t}\right)$ is:

$$
f_{, v}=0 .
$$

In terms of mobilities, Condition I reads:

$$
\lambda_{w, v}=0
$$

that is, the derivative of the water relative mobility with respect to gas saturation is zero. This condition is immediately satisfied because water mobility is identically zero along this edge.

We also require that the denominator of Equation (33) is nonzero:

$$
\nu_{2}-f_{, u} \neq 0
$$


Table 2. Summary of conditions along the OG edge

\begin{tabular}{crrl} 
Condition & Frac. flows & & Mobilities \\
\hline I & $f_{, v}=0$ & $\Leftrightarrow$ & $\lambda_{w, v}=0$ \\
II & $g_{, v}-f_{, u}>0$ & $\Leftrightarrow$ & $\lambda_{g, v}>\lambda_{w, u}+\lambda_{T, v} \frac{\lambda_{g}}{\lambda_{T}}$ \\
II at O & & & $\lambda_{g, v}>\lambda_{w, u}$ \\
II at G & & & $\lambda_{w, u}+\lambda_{o, v}=0$
\end{tabular}

Due to Equation (51), the eigenvalues take the following expressions along the OG edge:

$$
\nu_{1,2}=\left\{\begin{array}{l}
f_{, u} \\
g_{, v}
\end{array}\right.
$$

We impose that the system is strictly hyperbolic everywhere along the OG edge, excluding the $\mathrm{G}$ vertex. The condition of strict hyperbolicity, $\nu_{1}<\nu_{2}$, implies that (Condition II):

$$
H_{o g}:=g_{, v}-f_{, u}>0
$$

or, equivalently:

$$
\lambda_{g, v}>\lambda_{w, u}+\lambda_{T, v} \frac{\lambda_{g}}{\lambda_{T}}
$$

When we specialize Condition II at the $\mathrm{O}$ vertex, we obtain:

$$
\lambda_{g, v}>\lambda_{w, u}
$$

Equation (57) requires again that the gas relative permeability has a positive slope at its endpoint saturation. The $\mathrm{G}$ vertex is assumed to be an umbilic point, where the slow and fast characteristic speeds coincide, that is, $\nu_{1}=\nu_{2}$. When expressed in terms of relative mobilities, the condition reads:

$$
\lambda_{w, u}+\lambda_{o, v}=0
$$

The conditions at the OG edge are summarized in Table 2.

\subsubsection{Analysis along the WG edge.}

This edge corresponds to the line of zero reduced oil saturation, $v=1-u$. The analysis at the WG edge is complicated by the fact that it is a tilted 
line in the $(u, v)$-plane. The fast eigenvector will be parallel to the WG edge $\left(\boldsymbol{r}_{2}=[-1,1]^{t}\right)$ if:

$$
\nu_{2}-f_{, u}=-f_{, v} .
$$

Substituting the expression for the eigenvalues (Equation (31)) into the condition above, one arrives at:

$$
f_{, v}+g_{, v}=f_{, u}+g_{, u}
$$

In terms of mobilities, because the oil mobility is zero along the WG edge, Condition I reads:

$$
\lambda_{o, v}=\lambda_{o, u}
$$

that is, the derivatives of the oil relative mobility with respect to gas and water saturations are equal. As for the other two edges, this condition is identically satisfied by all models.

Due to Equation (60), the eigenvalues take the following expressions along the WG edge:

$$
\begin{aligned}
& \nu_{1}=g_{, v}+f_{, v} \\
& \nu_{2}=g_{, v}-g_{, u}
\end{aligned}
$$

The system is strictly hyperbolic everywhere along the WG edge $\left(\nu_{1}<\nu_{2}\right)$, excluding the $\mathrm{G}$ vertex, if:

$$
H_{w g}:=-g_{, u}-f_{, v}>0
$$

or, equivalently:

$$
\frac{\lambda_{w}}{\lambda_{T}}\left(\lambda_{g, v}-\lambda_{g, u}\right)+\frac{\lambda_{g}}{\lambda_{T}}\left(\lambda_{w, u}-\lambda_{w, v}\right)>-\lambda_{o, u} .
$$

The strict inequality at the $\mathrm{W}$ vertex reads:

$$
\lambda_{g, v}-\lambda_{g, u}>-\lambda_{o, u}
$$

and the equality at the $\mathrm{G}$ vertex (umbilic point) imposes that

$$
\lambda_{w, u}-\lambda_{w, v}=-\lambda_{o, u} .
$$


Table 3. Summary of conditions along the WG edge

\begin{tabular}{crll} 
Condition & Frac. flows & & Mobilities \\
\hline I & $f_{, v}+g_{, v}=f_{, u}+g_{, u}$ & $\Leftrightarrow$ & $\lambda_{o, v}=\lambda_{o, u}$ \\
II & $-g_{, u}-f_{, v}>0$ & $\Leftrightarrow$ & $\frac{\lambda_{w}}{\lambda_{T}}\left(\lambda_{g, v}-\lambda_{g, u}\right)$ \\
& & & $+\frac{\lambda_{g}}{\lambda_{T}}\left(\lambda_{w, u}-\lambda_{w, v}\right)>-\lambda_{o, u}$ \\
& & & $\lambda_{g, v}>-\lambda_{o, u}$ \\
II at W & & & $\lambda_{w, u}+\lambda_{o, v}=0$ \\
II at G & &
\end{tabular}

Substituting Condition I along all three edges (Equations (44), (52) and (61)) into (66)-(67) above, we obtain the conditions at the vertices in their final form:

$$
\begin{array}{ll}
\lambda_{g, v}>-\lambda_{o, u} & \text { at the } \mathrm{W} \text { vertex } \\
\lambda_{w, u}+\lambda_{o, v}=0 & \text { at the } \mathrm{G} \text { vertex. }
\end{array}
$$

In Table 3 we summarize the conditions at the WG edge.

Remark. The conditions expressed in Tables 1-3 are necessary conditions for strict hyperbolicity of the system of equations everywhere in the saturation triangle (with the exception of the $G$ vertex, which is an umbilic point). They are not sufficient.

\subsection{A simple model}

Our interest here reduces to presenting a simple model that satisfies the conditions above. A common practice in petroleum engineering [68,69] is to assume that relative permeabilities of the most and least wetting fluids (usually water and gas) depend only on their own saturation, whereas the relative permeability of the intermediate wetting fluid (usually oil) depends on all saturations. Although we do not defend this assumption in general, here we show that it is possible to obtain models which are strictly hyperbolic everywhere in the three-phase flow region. We take, for example:

$$
\begin{aligned}
\lambda_{w} & =\left(1 / \mu_{w}\right) u^{2}, \\
\lambda_{g} & =\left(1 / \mu_{g}\right)\left(\beta_{g} v+\left(1-\beta_{g}\right) v^{2}\right), \quad \beta_{g}>0 \\
\lambda_{o} & =\left(1 / \mu_{o}\right)(1-u-v)(1-u)(1-v) .
\end{aligned}
$$




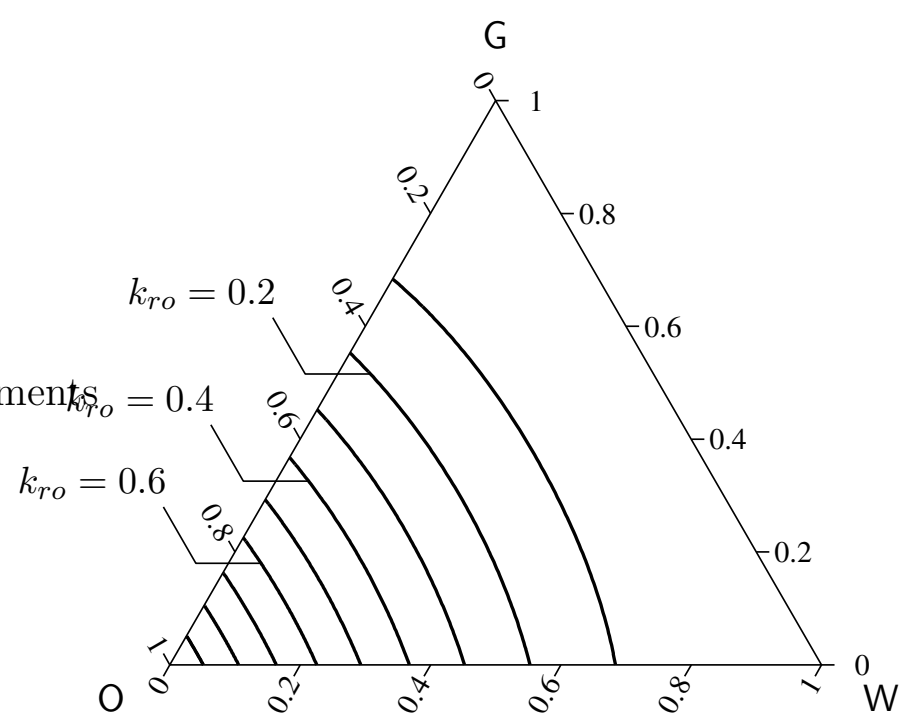

Figure 6. Oil isoperms for the simple model (70). For this particular case, oil isoperms are slightly convex towards the $\mathrm{O}$ corner.

The most important feature of the model is the positive derivative of the gas relative permeability function as it approaches zero. For the particular function used here, oil isoperms are slightly convex (Figure 6).

It is immediate to check that the relative mobilities (68)-(70) satisfy Condition I on all three edges. Whether Condition II is satisfied will depend, in general, on the values of the fluid viscosities and the endpoint-slope of the gas relative permeability.

\subsubsection{Analysis along the OW edge}

We need to study admissible values of the endpoint slope of the gas relative permeability, $\beta_{g}$, such that Equation (47) is satisfied for any given fluid viscosities. The derivatives of the fractional flow functions along the OW edge $(v=0)$ are as follows:

$$
\begin{aligned}
& f_{, u}=\frac{1}{\frac{u^{2}}{\mu_{w}}+\frac{(1-u)^{2}}{\mu_{o}}} \cdot 2 \frac{u(1-u)}{\mu_{o} u^{2}+\mu_{w}(1-u)^{2}}, \\
& g_{, v}=\frac{1}{\frac{u^{2}}{\mu_{w}}+\frac{(1-u)^{2}}{\mu_{o}}} \cdot \frac{\beta_{g}}{\mu_{g}} .
\end{aligned}
$$


Then, the condition for strict hyperbolicity along the OW edge reads:

$$
H_{o w}=g_{, v}-f_{, u}=\frac{1}{D(u)}\left[\frac{\beta_{g}}{\mu_{g}}-F(u)\right]>0
$$

where

$$
D(u):=\frac{1}{\frac{u^{2}}{\mu_{w}}+\frac{(1-u)^{2}}{\mu_{o}}}>0 \quad \forall u \in[0,1],
$$

and

$$
F(u):=2 \frac{u(1-u)}{\mu_{o} u^{2}+\mu_{w}(1-u)^{2}} .
$$

Defining $M:=\max _{0<u<1} F(u)$, Condition II on OW will be satisfied if

$$
\beta_{g}>\mu_{g} M
$$

Differentiating $F(u)$ with respect to $u$ and equating to zero, and after some algebraic manipulations, one obtains that $M=1 / \bar{\mu}$, where $\bar{\mu}:=\sqrt{\mu_{o} \mu_{w}}$ is the geometric mean of the water and oil viscosities. Finally, the condition for strict hyperbolicity on the OW edge is

$$
\beta_{g}>\frac{\mu_{g}}{\bar{\mu}}, \quad \bar{\mu}=\sqrt{\mu_{o} \mu_{w}}
$$

Despite the fact that Equation (77) is restricted to the simple model considered here, it is illuminating with regard to the required behavior for the relative permeability of the most nonwetting phase. Equation (77) expresses that there is a lower bound in the endpoint slope of the nonwetting phase relative permeability, if the three-phase flow model is to be strictly hyperbolic. This threshold is proportional to the ratio between the viscosity of the nonwetting phase and the average viscosity of the other two phases. This is perfectly consistent with the expected behavior in real displacements:

1. If the viscosity of the most nonwetting phase is very small compared to that of the other phases, one expects fingering (unstable displacement) when the nonwetting phase is injected. This results in an early breaktrough with small abrupt changes between having no flow and some flow at the outlet. This implies a small value of the endpoint slope of the relative permeability, $\beta_{g}$. 
2. On the other hand, if the viscosity of the nonwetting phase is comparable with that of the other phases in the medium, the displacement of that phase will be stable, and will cause that the transition between zero flow and nonzero flow at the outlet will be more drastic, resulting in a larger value of $\beta_{g}$.

\subsubsection{Analysis along the OG edge}

We now study if the relative permeability model satisfies Condition II along the OG edge of the saturation triangle $(u=0)$. Along this edge, the derivatives of the fractional flow functions involved in Equation (55) are:

$$
\begin{aligned}
& f_{, u}=0, \\
& g_{, v}=\frac{1}{\mu_{g} \mu_{o}} \frac{1-v}{\left(\frac{\beta_{g} v+\left(1-\beta_{g}\right) v^{2}}{\mu_{g}}+\frac{(1-v)^{2}}{\mu_{o}}\right)^{2}} \cdot\left(\beta_{g}+\left(2-\beta_{g}\right) v\right) .
\end{aligned}
$$

For strict hyperbolicity along the OG edge (excluding the G vertex), we require:

$$
H_{o g}=g_{, v}-f_{, u}=\frac{1}{\mu_{g} \mu_{o}} \frac{1-v}{\left(\frac{\beta_{g} v+\left(1-\beta_{g}\right) v^{2}}{\mu_{g}}+\frac{(1-v)^{2}}{\mu_{o}}\right)^{2}} \cdot\left(\beta_{g}+\left(2-\beta_{g}\right) v\right)>0,
$$

which is always satisfied for all $v \in[0,1)$, as long as the endpoint slope $\beta_{g}>0$. At the $\mathrm{G}$ vertex $(u=0, v=1)$, we obtain $H_{o g}=0$, so this point is an umbilic point, as required.

\subsubsection{Analysis along the WG edge}

We perform now the same analysis on the edge of zero reduced oil saturation, $u=1-v$. The expressions of the fractional flow derivatives in Equation (64) are:

$$
\begin{aligned}
& f_{, v}=\frac{-1}{\left(\frac{\beta_{g} v+\left(1-\beta_{g}\right) v^{2}}{\mu_{g}}+\frac{(1-v)^{2}}{\mu_{w}}\right)^{2}} \cdot\left[\frac{\beta_{g}+2\left(1-\beta_{g}\right) v}{\mu_{g}}+\frac{v(1-v)}{\mu_{o}}\right] \cdot \frac{(1-v)^{2}}{\mu_{w}}, \\
& g_{, u}=\frac{-1}{\left(\frac{\beta_{g} v+\left(1-\beta_{g}\right) v^{2}}{\mu_{g}}+\frac{(1-v)^{2}}{\mu_{w}}\right)^{2}} \cdot\left[\frac{2(1-v)}{\mu_{w}}-\frac{v(1-v)}{\mu_{o}}\right] \cdot \frac{\beta_{g} v+\left(1-\beta_{g}\right) v^{2}}{\mu_{g}} .
\end{aligned}
$$


The condition for strict hyperbolicity along this edge (not including the G vertex) is

$$
H_{w g}=-g_{, u}-f_{, v}=\frac{1}{E(v)}\left[\frac{C_{w g}(v)}{\mu_{w} \mu_{g}}+\frac{C_{o g}(v)}{\mu_{o} \mu_{g}}+\frac{C_{w o}(v)}{\mu_{w} \mu_{o}}\right]>0
$$

where

$$
\begin{aligned}
E(v) & =\left(\frac{\beta_{g} v+\left(1-\beta_{g}\right) v^{2}}{\mu_{g}}+\frac{(1-v)^{2}}{\mu_{w}}\right)^{2}, \\
C_{w g}(v) & =\left(2-\left(2-\beta_{g}\right)(1-v)\right)(1-v), \\
C_{o g}(v) & =-\left(1-\left(1-\beta_{g}\right)(1-v)\right) v^{2}(1-v), \\
C_{o w}(v) & =-v(1-v)^{3} .
\end{aligned}
$$

At the $\mathrm{G}$ vertex, corresponding to $v=1$, it is clear that $H_{w g}=0$. On the other hand, it is not easy to infer the conditions on the fluid viscosities and the endpoint slope $\beta_{g}$ such that the strict inequality (83) is satisfied on the entire edge. It is possible, however, to identify the conditions for strict hyperbolicity along this edge near the $G$ vertex.

Let $u=\varepsilon, v=1-\varepsilon$ with $\varepsilon \rightarrow 0$, that is, a state on the WG edge near the $\mathrm{G}$ corner. The first-order Taylor expansion of $H_{w g}$ about $\varepsilon=0$ is

$$
H_{w g}=\mu_{g}\left[\frac{2}{\mu_{w}}-\frac{1}{\mu_{o}}\right] \varepsilon+O\left(\varepsilon^{2}\right) .
$$

Therefore, for $H_{w g}>0$ in the neighborhood of the $\mathrm{G}$ corner, we obtain the condition

$$
\mu_{w}<2 \mu_{o}
$$

This imposes an additional restriction (not obvious to anticipate) on the values of the fluid viscosities, if one wants the relative permeability model (68)(70) to yield a strictly hyperbolic system.

\subsubsection{Summary of conditions for strict hyperbolicity of the model}

We have arrived at the conclusion that the very simple relative permeability model (68)-(70) may yield strictly hyperbolic behavior everywhere in the saturation triangle, except at the vertex of $100 \%$ reduced gas saturation, 


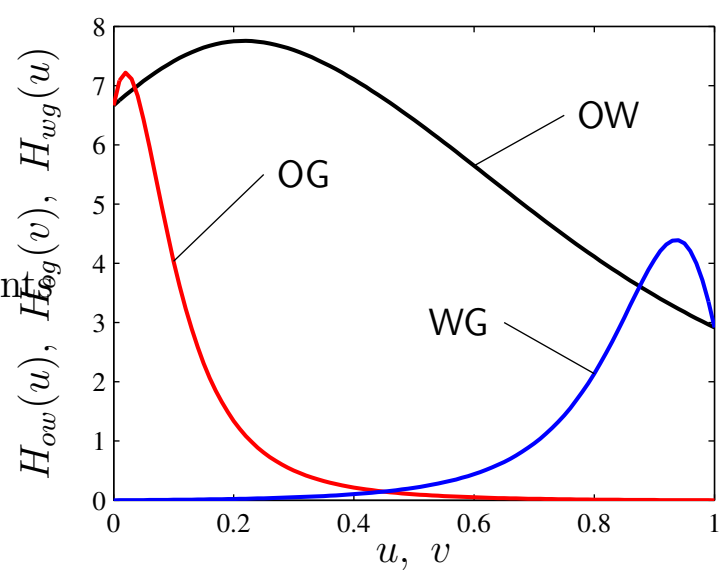

Figure 7. Strict hyperbolicity on edges of the saturation triangle (Condition II) requires that all three functions $H_{o w}(u), H_{o g}(v)$, and $H_{w g}(u)$ are positive everywhere.

which is an umbilic point. The only two conditions that the parameters of the model need to satisfy are:

$$
\begin{aligned}
\beta_{g} & >\frac{\mu_{g}}{\sqrt{\mu_{o} \mu_{w}}}, \\
\mu_{w} & <2 \mu_{o} .
\end{aligned}
$$

For illustrative purposes, we take reasonable values of the viscosities:

$$
\mu_{w}=0.875, \quad \mu_{g}=0.03, \quad \mu_{o}=2 \mathrm{cp},
$$

and a small value of the endpoint slope: $\beta_{g}=0.1$. These values of the parameters satisfy the two conditions above. In Figure 7 we represent graphically the functions $H_{o w}(u)$ along OW, $H_{o g}(v)$ along OG, and $H_{w g}(u)$ along WG. Inequalities (47), (55), and (64) are satisfied (and the system is strictly hyperbolic) if all three curves are positive everywhere. The curves for the OG edge and the WG edge reach a zero value for $v=1$ and $u=0$, respectively, so that the $G$ vertex is an umbilic point.

\section{Validation with experimental data}

In Section 3 we derived the necessary conditions that must be satisfied by the relative permeability functions, if the system of equations describing three- 
phase flow is to be strictly hyperbolic everywhere inside the saturation triangle. The essential requirement for strict hyperbolicity turns out to be that the relative permeability of gas (the most nonwetting phase) must have a positive derivative with respect to its own saturation, at the edge of zero reduced gas saturation (Equation (48)). In this section we verify how realistic this condition is, by means of comparison with experimental data. To this end, we use Oak's steady-state experiments $[54,56]$, which are arguably the most reliable and best-known data set available. The fact that we use steady-state relative permeability data in a dynamic fluid displacement model ought to be of little consequence because the relative permeabilities measured with many different methods are similar $[16,35,57,73]$.

The data set consists of over 1800 two-phase and three-phase relative permeability measurements, obtained using a fully automated steady-state method. Three fired Berea sandstone cores were employed, with absolute permeabilities of 200 md (Sample 6), 800 md (Sample 14), and 1000 md (Sample 13). Water, oil, and gas viscosities were 1.06, 1.77, and $0.0187 \mathrm{cp}$, respectively. The study includes over 30 combinations of rock and fluid systems and saturation histories. A complete description of the experimental apparatus and procedure is given in the original references $[54,56]$.

\subsection{Description of the "endpoint-slope" analysis}

We are interested in the qualitative behavior of the relative permeability of each phase in the region of low reduced saturation of that phase. More precisely, we want to determine whether the relative permeability of a phase, when expressed as a function of its own saturation only, takes off with a zero or a positive slope. This fundamentally different behavior is shown schematically in Figure 8.

The steps involved in our "endpoint-slope" analysis of Oak's relative permeability data are described below:

1. Select an experiment. An experiment consists of several (sometimes dozens) relative permeability measurements.

2. Regardless of the type of experiment (two-phase, or three-phase; drainage or imbibition), tabulate the relative permeability of a phase against its own saturation. 


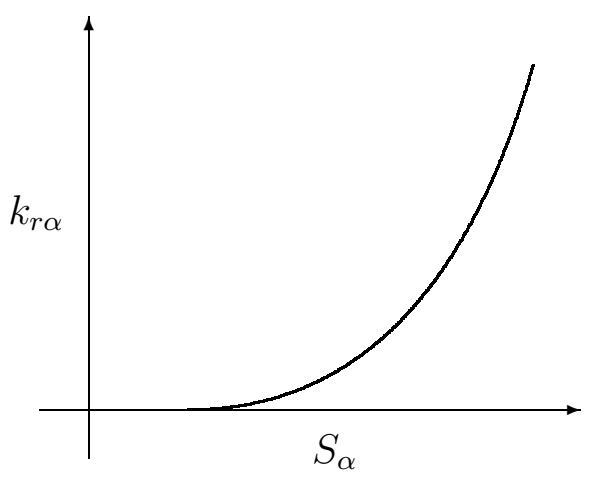

(a) Zero-slope behavior

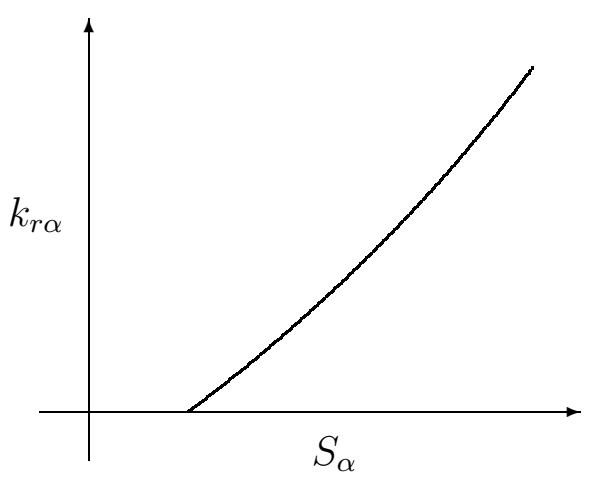

(b) Positive-slope behavior

Figure 8. Markedly different qualitative behavior of the slope of the relative permeability of a phase, in the region near the "immobile" saturation of that phase: (a) zero-slope, and (b) positive slope.

3. For each phase $\alpha$, identify a maximum saturation $S_{\alpha, \max }$ that defines the range of saturations to be used in the analysis, and use only the data points for saturations $S_{\alpha}<S_{\alpha, \max }$. This range should be small enough to be considered close to the immobile saturation, but should have a sufficient number of data points to indicate a trend.

4. For each phase $\alpha$, fit the power-law expression

$$
k_{r \alpha}=C_{\alpha}\left(S_{\alpha}-S_{\alpha i}\right)^{m_{\alpha}}, \quad S_{\alpha}<S_{\alpha, \max },
$$

using a least squares procedure [12], with the following constraints:

$$
C_{\alpha}>0, \quad S_{\alpha i} \geq 0, \quad m_{\alpha} \geq 1 .
$$

Out of the three parameters to be optimized, the most relevant for our discussion is the exponent $m_{\alpha}$. A value of 1 or close to 1 is indicative of a linear behavior of the relative permeability and, thus, a positive slope at the endpoint saturation. On the other hand, an exponent larger than 2 suggests that the relative permeability will approach a zero value (at the endpoint saturation) with zero slope.

Here we present the results of the analysis of four representative experiments, to show how well the condition of positive endpoint slope for the gas relative permeability is satisfied by actual data. 


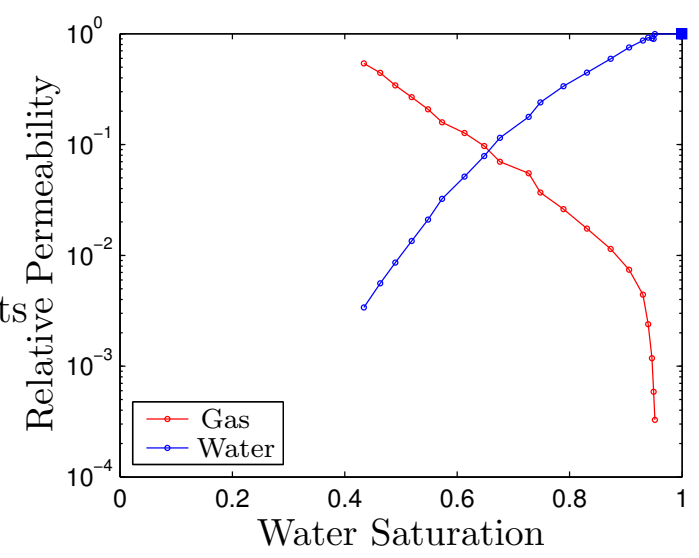

Figure 9. Relative permeability curves of water and gas for the twophase drainage experiment (Sample 13, Experiment 16a of Oak's dataset). The solid square mark ( $\mathbf{\square})$ indicates the initial saturation state of the core.

\subsection{Two-phase flow experiments}

The selected data come from two consecutive experiments, performed on a fired Berea sandstone core of about 1000 md (Sample 13), using water and gas as the wetting and the nonwetting phase, respectively.

\subsubsection{Primary drainage experiment}

The first experiment (Experiment 16a) corresponds to a drainage process, where gas is injected into an initially water-filled core. This is done through a sequence of steady states: water and gas are injected at constant rates and, when steady state is achieved, the relative permeabilities and average saturations are measured; then, the ratio of gas/water flow rates is increased, the system reaches a new steady state, and the process continues. The relative permeability curves for this experiment are plotted in Figure 9 in semi-log scale.

The results of the power-law fitting of the data from the two-phase drainage experiment are presented in Table 4. The most important observation is the essential difference in the value of the exponent $m_{\alpha}$ for the wetting phase $\left(m_{w} \approx 8\right)$ and the nonwetting phase $\left(m_{g} \approx 1.1\right)$. The actual fit of the relative permeability data of water and gas is shown graphically in Figure 10. From this figure it is apparent that the water relative permeability reaches a 
Table 4. Parameters of the power-law fitting for the two-phase drainage experiment. Note the major difference in the value of the exponent $m_{\alpha}$ for water and gas.

\begin{tabular}{ccc} 
& Water & Gas \\
\hline$S_{\alpha, \max }$ & 0.7000 & 0.2500 \\
$C_{\alpha}$ & 2.6362 & 0.1902 \\
$S_{\alpha i}$ & 0.0000 & 0.0404 \\
$m_{\alpha}$ & $\mathbf{8 . 0 2 8 2}$ & $\mathbf{1 . 1 3 7 7}$ \\
\hline
\end{tabular}

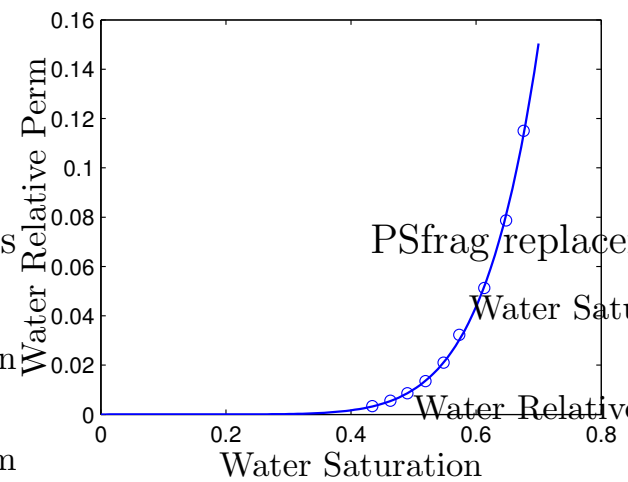

(a) Water relative permeability

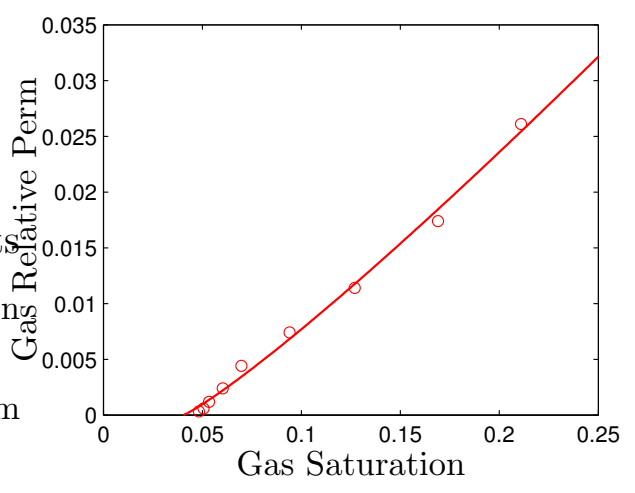

(b) Gas relative permeability

Figure 10. Behavior of the relative permeability as a function of its own saturation in the neighborhood of the "endpoint saturation", for the two-phase drainage experiment: (a) the water relative permeability curve shows a high-order behavior and, therefore, a zero slope near the connate water saturation; (b) on the other hand, the gas relative permeability curve displays an almostlinear relation against gas saturation, which can be assimilated to a nonzero slope near the critical gas saturation.

value of zero with a zero value of the slope, whereas the slope of the gas relative permeability curve is finite and positive. This experimental observation is in agreement with the key condition (48) proposed in this paper. 


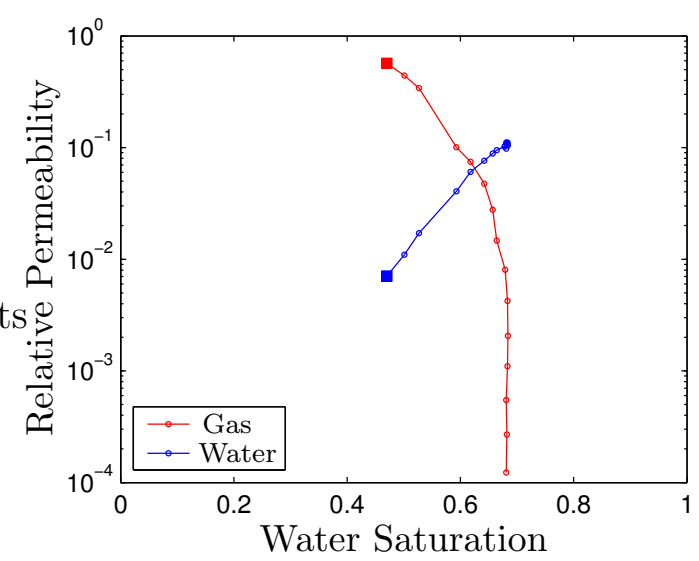

Figure 11. Relative permeability curves of water and gas for the two-phase imbibition experiment (Sample 13, Experiment 16b of Oak's dataset). The solid square mark ( $\mathbf{\square})$ indicates the saturation state of the core after primary drainage.

\subsubsection{Secondary imbibition experiment}

The data analyzed here (Sample 13, Experiment 16b) correspond to the imbibition process following the primary drainage experiment described in the previous paragraph. As before, the experiment involves a sequence of steady states, except that now, when a steady state is achieved, the ratio of gas/water flow rates is decreased. The relative permeability curves obtained in this way are plotted in semi-log scale in Figure 11.

The results of the power-law fitting to the data are presented in Table 5 . Once again, the values of the exponent $m_{\alpha}$ for the wetting phase $\left(m_{w} \approx 4\right)$ and the nonwetting phase $\left(m_{g}=1\right)$ are fundamentally different. The actual fit to the relative permeability data of water and gas is shown graphically in Figure 12, and the same comments as in the drainage experiment follow: a zero slope of the water relative permeability, and a positive slope of the gas relative permeability near their respective immobile saturations.

\subsection{Three-phase flow experiments}

We repeat the same analysis for experiments involving simultaneous flow of three fluids: water, oil, and gas. Water is the most wetting phase, and gas is the least wetting. We present the results of two experiments: one simulating 
Table 5. Parameters of the power-law fitting for the two-phase imbibition experiment.

\begin{tabular}{ccc} 
& Water & Gas \\
\hline$S_{\alpha, \max }$ & 0.6700 & 0.4500 \\
$C_{\alpha}$ & 3.9379 & 1.2051 \\
$S_{\alpha i}$ & 0.2785 & 0.3212 \\
$m_{\alpha}$ & $\mathbf{3 . 9 0 3 0}$ & $\mathbf{1 . 0 0 0 0}$ \\
\hline
\end{tabular}

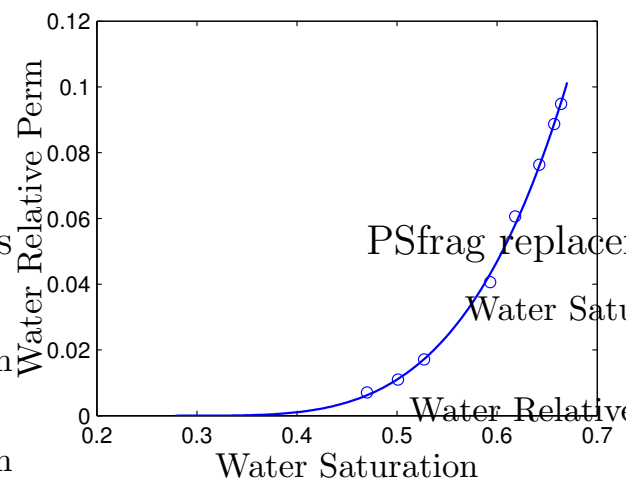

(a) Water relative permeability

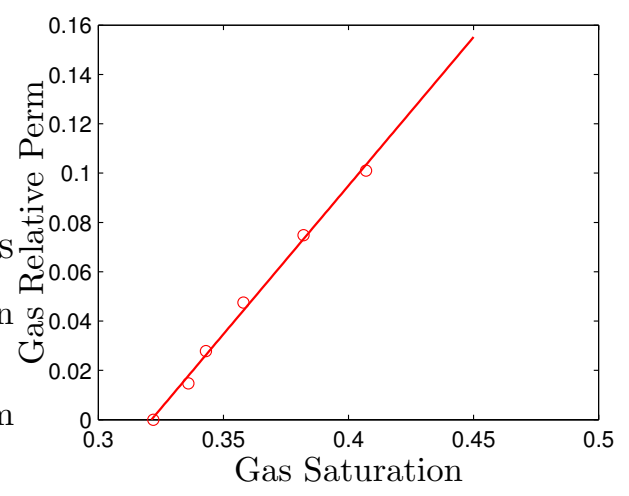

(b) Gas relative permeability

Figure 12. Behavior of the relative permeability as a function of its own saturation in the neighborhood of the "endpoint saturation", for the two-phase imbibition experiment. Remarks in Figure 10 apply. 


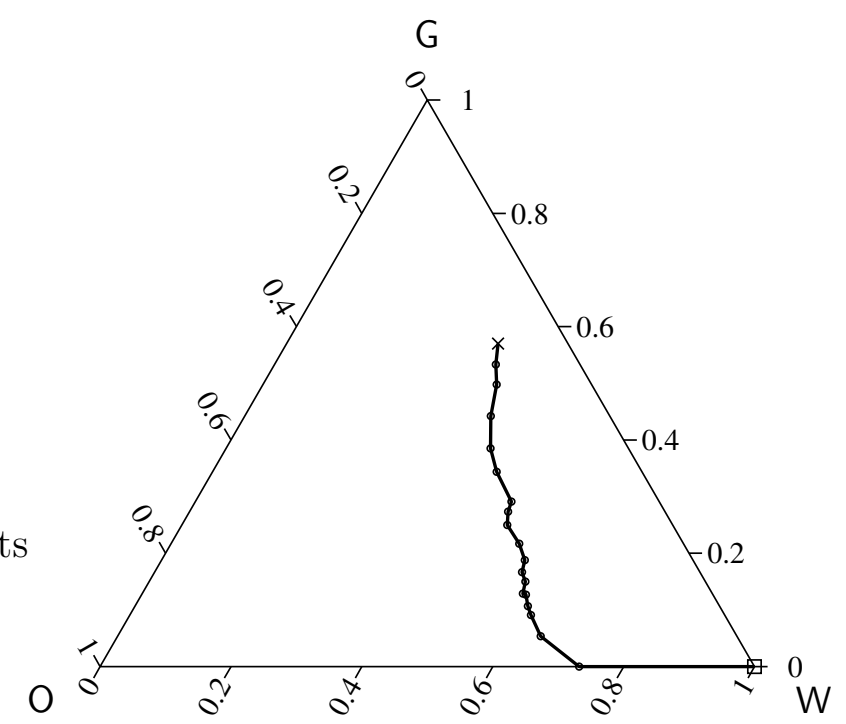

Figure 13. Saturation path for the drainage-dominated three-phase relative permeability experiment (Sample 6, Experiment 15a of Oak's dataset). The square mark $(\square)$ indicates the saturation state of the core at the beginning of the experiment (initially water-filled).

gas injection, and the other characteristic of waterflooding.

\subsubsection{Drainage-dominated experiment}

The first of the three-phase flow experiments consists in a sequence of steady states of increasing average gas saturation. The jump in saturations from one steady-state to the next is achieved by appropriately modifying the flow rates at which each fluid is injected into the core. In particular, the ratio of gas/water flow rates is increased, while the water/oil ratio is held constant. The resulting saturation path for this experiment is shown on a ternary diagram in Figure 13.

In Table 6 we present the parameters of the power-law fit for the water and gas phases. In accordance with the two-phase flow results, we observe a high value of the exponent $\left(m_{w} \approx 3\right)$ for water, and a value close to one $\left(m_{g} \approx 1.2\right)$ for gas. The obvious interpretation is that the water relative permeability curve reaches zero with a zero-value of the slope, whereas the 
Table 6. Parameters of the power-law fitting for the three-phase drainagedominated experiment.

\begin{tabular}{ccc} 
& Water & Gas \\
\hline$S_{\alpha, \max }$ & 0.4840 & 0.4000 \\
$C_{\alpha}$ & 0.1065 & 0.7162 \\
$S_{\alpha i}$ & 0.2092 & 0.0941 \\
$m_{\alpha}$ & $\mathbf{2 . 8 6 2 3}$ & $\mathbf{1 . 2 5 5 2}$ \\
\hline
\end{tabular}

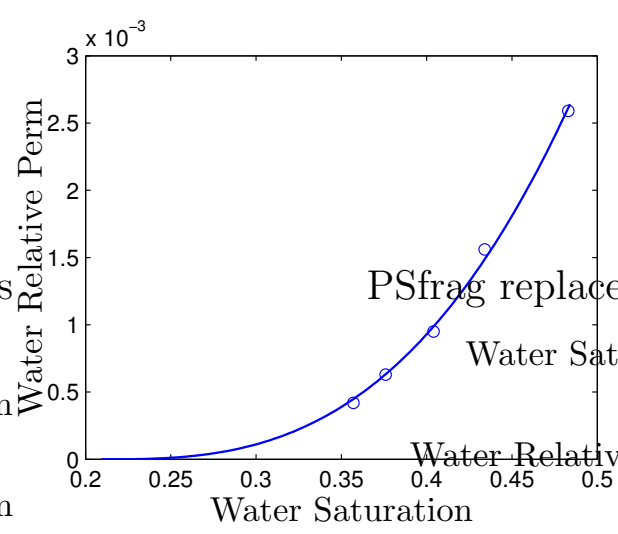

(a) Water relative permeability

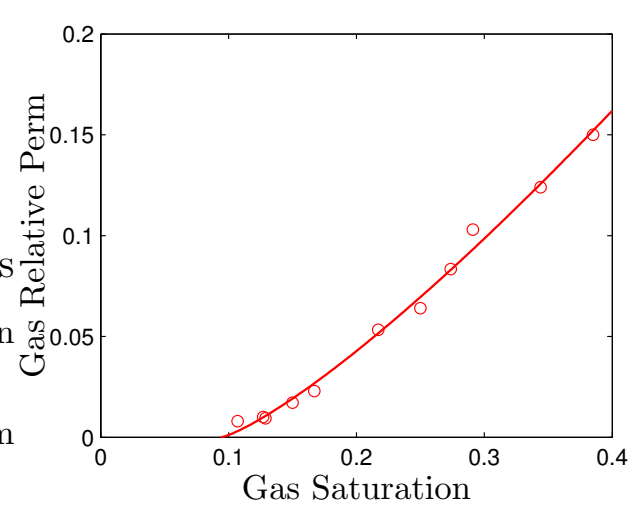

(b) Gas relative permeability

Figure 14. Behavior of the relative permeability as a function of its own saturation in the neighborhood of the "endpoint saturation", for the three-phase drainage-dominated experiment. Resemblance of actual data to the conceptual picture of Figure 8 is apparent.

slope is positive for the gas relative permeability (Figure 14).

\subsubsection{Imbibition-dominated experiment}

Our last example is a three-phase flow experiment, in which fluids are injected in such a way that steady states of increasing water saturation are obtained. This sequence is achieved by decreasing the ratio of gas/water flow rates, while keeping constant the water/oil ratio. In Figure 15 we depict the saturation path for this experiment, whose starting point is the saturation state obtained at the end of drainage-dominated process analyzed before.

Table 7 has the numeric values of the power-law fit, and Figure 16 shows the experimental data and the fitted relative permeability curves. The same 


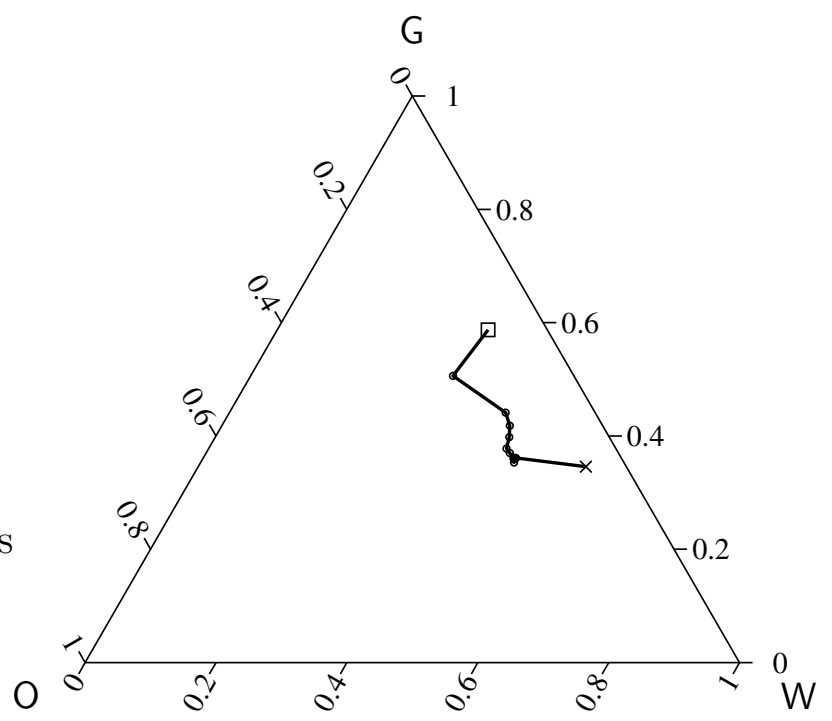

Figure 15. Saturation path for the imbibition-dominated three-phase relative permeability experiment (Sample 6, Experiment 15b of Oak's dataset). The square mark $(\square)$ indicates the saturation state of the core at the end of the drainage-dominated experiment (gas injection).

Table 7. Parameters of the power-law fitting for the three-phase imbibitiondominated experiment.

\begin{tabular}{ccc} 
& Water & Gas \\
\hline$S_{\alpha, \max }$ & 0.5000 & 0.6000 \\
$C_{\alpha}$ & 0.4737 & 2.1985 \\
$S_{\alpha i}$ & 0.2569 & 0.3530 \\
$m_{\alpha}$ & $\mathbf{3 . 2 8 0 4}$ & $\mathbf{1 . 0 0 0 0}$ \\
\hline
\end{tabular}

qualitative behavior as that of the previous examples is observed $\left(m_{w} \approx 3\right.$, $\left.m_{g}=1\right)$.

Remark. The particular examples presented herein are representative of more than one hundred experiments in Oak's dataset. Experimental data seems to corroborate the fundamental requirement for strict hyperbolicity of the model, that is, a positive slope of the relative permeability of the most nonwetting phase near its immobile saturation. 


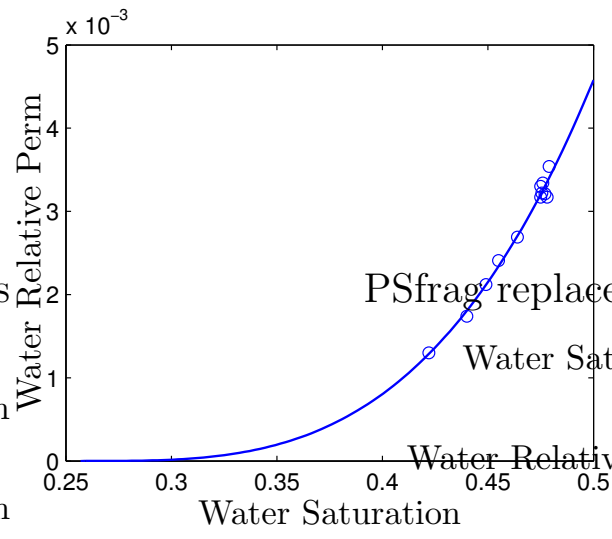

(a) Water relative permeability

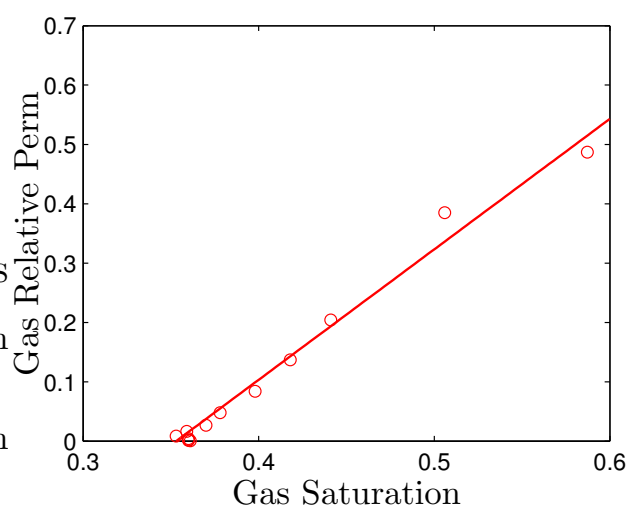

(b) Gas relative permeability

Figure 16. Behavior of the relative permeability as a function of its own saturation in the neighborhood of the "endpoint saturation", for the three-phase imbibition-dominated experiment.

\section{Conclusions and future work}

Traditional formulations of three-phase flow in porous media employ the usual extension of Darcy's equation to model fluid fluxes. Within this framework, it was believed that elliptic regions were unavoidable when generic relative permeability functions were used in models of one-dimensional immiscible incompressible three-phase flow. This conclusion was inferred after a particular behavior of the relative permeabilities along the edges of the saturation triangle was assumed.

In this paper we show it is possible to identify conditions which the relative permeability functions must satisfy for the system of equations to be strictly hyperbolic everywhere in the saturation triangle. By means of a specific example, we suggest how strict hyperbolicity may be invoked to impose constraints on the parameters of the relative permeability model. It turns out that the fundamental requirement is a finite positive slope of the gas relative permeability at the saturation where gas becomes mobile. This condition is consistent with a pore-scale description of multiphase flow and, as shown in this paper, is also supported by experimental relative permeability data.

This important result is restricted to the case when gravitational effects are not accounted for, which is sensible only when the gravity number is 
small, or when flow is horizontal. It is possible, however, to extend this analysis to the case when flow is not horizontal and gravity is included, by allowing that relative permeabilities may vary with the gravity number [38]. This dependence is physically reasonable, and can be justified in terms of the stability of the displacement of one fluid by another.

We admit that we still do not have a definite argument in favor or against the presence of elliptic regions in the saturation space, although we find good reasons to believe they are nothing else than mathematical artifacts of an incorrect mathematical model. The implications of having elliptic character in models of three-phase flow are discussed more fully elsewhere [37, 39]. Our current view is that one should use these hints to develop new relative permeability models, or an improved description altogether of three-phase flow in porous media.

\section{ACKNOWLEDGEMENTS}

We are grateful to Dr. Dmitriy Silin for numerous insightful suggestions and his careful review of the manuscript. We also thank Prof. Martin Blunt for his comments and the vigorous discussion on elliptic regions, and for providing an electronic version of Oak's relative permeability data. This work was supported by the Laboratory Directed Research and Development Program of Lawrence Berkeley National Laboratory under the Department of Energy Contract No. DE-AC0376SF00098. Funding provided by Barrié de la Maza, Jane Lewis, and Repsol-YPF fellowships, awarded to the first author, is also gratefully acknowledged.

\section{Unit conversion factors}

\begin{tabular}{l}
1 centipoise $(\mathrm{cp})=10^{-3} \mathrm{~Pa} \mathrm{~s}$ \\
1 milidarcy $(\mathrm{md})=0.9869233 \times 10^{-15} \mathrm{~m}^{2}$ \\
\hline
\end{tabular}

\section{References}

[1] S. Axler. Linear Algebra Done Right. Undergraduate Texts in Mathematics. Springer-Verlag, New York, 1996.

[2] A. V. Azevedo and D. Marchesin. Multiple viscous profile Riemann solutions in mixed elliptic-hyperbolic models for flow in porous media. 
In B. L. Keyfitz and M. Shearer, editors, Nonlinear Evolution Equations that Change Type, volume 27 of The IMA Volumes in Mathematics and its Applications, pages 1-17. Springer-Verlag, New York, 1990.

[3] A. V. Azevedo and D. Marchesin. Multiple viscous solutions for systems of conservation laws. Trans. Amer. Math. Soc., 347(8):3061-3077, 1995.

[4] K. Aziz and A. Settari. Petroleum Reservoir Simulation. Elsevier, London, 1979.

[5] G. I. Barenblatt, V. M. Entov, and V. M. Ryzhik. Theory of Fluid Flows through Natural Rocks, volume 3 of Theory and Applications of Transport in Porous Media. Kluwer, Dordrecht, 1990. Expanded and revised edition of the original in Russian Dvizhenie zhidkostei $i$ gazov $v$ prirodnykh plastakh, Nedra Publishers, 1984.

[6] J. Bear. Dynamics of Fluids in Porous Media. Environmental Science Series. Elsevier, New York, 1972. Reprinted with corrections, Dover, New York, 1988.

[7] J. B. Bell, J. A. Trangenstein, and G. R. Shubin. Conservation laws of mixed type describing three-phase flow in porous media. SIAM J. Appl. Math., 46(6):1000-1017, 1986.

[8] H. G. Botset. Flow of gas-liquid mixtures through consolidated sands. Petrol. Trans. AIME, 136:91-105, 1940.

[9] G. Chavent and J. Jaffré. Mathematical Models and Finite Elements for Reservoir Simulation, volume 17 of Studies in Mathematics and its Applications. Elsevier, North-Holland, 1986.

[10] G. Chavent, J. Jaffré, and S. Jan-Jégou. Estimation of relative permeabilities in three-phase flow in porous media. Inverse Problems, 15:3339, 1999.

[11] E. A. Coddington and N. Levinson. Theory of Ordinary Differential Equations. McGraw Hill, New York, 1955.

[12] T. Coleman, M. A. Branch, and A. Grace. MATLAB Optimization Toolbox v. 2.1. The MathWorks, Natick, MA, 2000. 
[13] R. Courant and K. O. Friedrichs. Supersonic Flow and Shock Waves. John Wiley \& Sons, New York, 1948.

[14] C. M. Dafermos. Hyperbolic Conservation Laws in Continuum Physics, volume 325 of A Series of Comprehensive Studies in Mathematics. Springer-Verlag, Berlin, 2000.

[15] D. A. DiCarlo, A. Sahni, and M. J. Blunt. The effect of wettability on three-phase relative permeability. Transp. Porous Media, 39:347-366, 2000 .

[16] D. A. Éfros. Determination of relative permeabilities and distribution functions in the displacement of oil by water [in Russian]. Dokl. Akad. Nauk SSSR, 110(5):746, 1956.

[17] A. H. Falls and W. M. Schulte. Features of three component, three phase displacement in porous media. SPERE, 7(4):426-432, November 1992.

[18] A. H. Falls and W. M. Schulte. Theory of three component, three phase displacement in porous media. SPERE, 7(3):377-384, August 1992.

[19] F. J. Fayers. Extension of Stone's method I and conditions for real characteristics in three-phase flow. In SPE 62nd Annual Technical Conference and Exhibition, Dallas, TX, September 27-30, 1987. (SPE 16965).

[20] F. J. Fayers and J. D. Matthews. Evaluation of normalized Stone's methods for estimating three-phase relative permeabilities. SPEJ, 24(2):224232, April 1984. Petrol. Trans. AIME, 277.

[21] T. M. Geffens, W. W. Owens, D. R. Parrish, and R. A. Morse. Experimental investigation of factors affecting laboratory relative permeability measurements. Petrol. Trans. AIME, 192:99-110, 1951.

[22] W. G. Gray and S. M. Hassanizadeh. Macroscale continuum mechanics for multiphase porous-media flow including phases, interfaces, common lines and common points. Adv. Water Resour., 21:261-281, 1998.

[23] R. E. Guzmán and F. J. Fayers. Mathematical properties of three-phase flow equations. SPEJ, 2(3):291, September 1997.

[24] R. E. Guzmán and F. J. Fayers. Solution to the three-phase flow Buckley-Leverett problem. SPEJ, 2(3):301, September 1997. 
[25] S. M. Hassanizadeh. Derivation of basic equations of mass transport in porous media, Part 2. Generalized Darcy's and Fick's laws. Adv. Water Resour., 9:207-222, 1986.

[26] S. M. Hassanizadeh and W. G. Gray. Mechanics and thermodynamics of multiphase flow in porous media including interphase boundaries. Adv. Water Resour., 13(4):169-186, 1990.

[27] S. M. Hassanizadeh and W. G. Gray. Toward an improved description of the physics of two-phase flow. Adv. Water Resour., 16(1):53-67, 1993.

[28] H. Holden. On the Riemann problem for a prototype of a mixed type conservation law. Comm. Pure Appl. Math., 40(2):229-264, 1987.

[29] H. Holden and L. Holden. On the Riemann problem for a prototype of a mixed type conservation law, II. In W. B. Lindquist, editor, Current Progress in Hyperbolic Systems: Riemann Problems and Computations, volume 100 of Contemporary Mathematics, pages 331-367. American Mathematical Society, Providence, RI, 1989.

[30] H. Holden, L. Holden, and N. H. Risebro. Some qualitative properties of $2 \times 2$ systems of conservation laws of mixed type. In B. L. Keyfitz and M. Shearer, editors, Nonlinear Evolution Equations that Change Type, volume 27 of The IMA Volumes in Mathematics and its Applications, pages 67-78. Springer-Verlag, New York, 1990.

[31] L. Holden. On the strict hyperbolicity of the Buckley-Leverett equations for three-phase flow in a porous medium. SIAM J. Appl. Math., 50(3):667-682, 1990.

[32] E. Isaacson, D. Marchesin, and B. Plohr. Transitional shock waves. In W. B. Lindquist, editor, Current Progress in Hyperbolic Systems: Riemann Problems and Computations, volume 100 of Contemporary Mathematics, pages 125-145. American Mathematical Society, Providence, RI, 1989.

[33] E. Isaacson, D. Marchesin, B. Plohr, and J. B. Temple. Multiphase flow models with singular Riemann problems. Mat. Aplic. Comp., 11(2):147166, 1992. 
R. Juanes and T. W. Patzek: Strictly hyperbolic models of three-phase flow

[34] M. D. Jackson and M. J. Blunt. Elliptic regions and stable solutions for three-phase flow in porous media. Transp. Porous Media, 48:249-269, 2002.

[35] E. F. Johnson, D. P. Bossler, and V. O. Naumann. Calculation of relative permeability from displacement experiments. Petrol. Trans. AIME, 216:370-372, 1959 .

[36] P. J. Hicks Jr. and A. S. Grader. Simulation of three-phase displacement experiments. Transp. Porous Media, 24:221-245, 1996.

[37] R. Juanes. Mathematical and numerical modeling of multiphase flow in porous media. PhD Dissertation, University of California at Berkeley, December 2002. (In preparation).

[38] R. Juanes and T. W. Patzek. Strictly hyperbolic models of three-phase flow with gravity. (In preparation).

[39] R. Juanes, D. B. Silin, and T. W. Patzek. On elliptic regions in models of three-phase flow through permeable rocks. (In preparation).

[40] B. L. Keyfitz. Shocks near the sonic line: a comparison between steady and unsteady models for change of type. In B. L. Keyfitz and M. Shearer, editors, Nonlinear Evolution Equations that Change Type, volume 27 of The IMA Volumes in Mathematics and its Applications, pages 89-106. Springer-Verlag, New York, 1990.

[41] C. S. Land. Calculation of imbibition relative permeability for two- and three-phase flow from rock properties. SPEJ, 8(2):149-156, June 1968. Petrol. Trans. AIME, 243.

[42] P. D. Lax. Hyperbolic systems of conservation laws, II. Comm. Pure Appl. Math., 10:537-566, 1957.

[43] R. J. Lenhard and J. C. Parker. A model for hysteretic constitutive relations governing multiphase flow, 2. Permeability-saturation relations. Water Resour. Res., 23(12):2197-2206, 1987.

[44] R. Lenormand. Pattern growth and fluid displacements through porous media. Physica A, 140:114-123, 1986. 
[45] R. Lenormand, E. Touboul, and C. Zarcone. Numerical models and experiments on immiscible displacements in porous media. J. Fluid Mech., 189:165-187, 1988.

[46] M. C. Leverett and W. B. Lewis. Steady flow of gas-oil-water mixtures through unconsolidated sands. Petrol. Trans. AIME, 142:107-116, 1941.

[47] T.-P. Liu. The Riemann problem for general $2 \times 2$ conservation laws. Trans. Amer. Math. Soc., 199:89-112, 1974.

[48] T.-P. Liu. The Riemann problem for general systems of conservation laws. J. Differential Equations, 18:218-234, 1975.

[49] D. Marchesin and H. B. Medeiros. A note on the stability of eigenvalue degeneracy in nonlinear conservation laws of multiphase flow. In W. B. Lindquist, editor, Current Progress in Hyperbolic Systems: Riemann Problems and Computations, volume 100 of Contemporary Mathematics, pages 215-224. American Mathematical Society, Providence, RI, 1989.

[50] D. Marchesin and B. J. Plohr. Wave structure in WAG recovery. SPEJ, 6(2):209-219, June 2001.

[51] C. T. Miller, G. Christakos, P. T. Imhoff, J. F. McBride, J. A. Pedit, and J. A. Trangenstein. Multiphase flow and transport modeling in heterogeneous porous media: challenges and approaches. Adv. Water Resour., 21(2):77-120, 1998.

[52] M. Muskat. Physical Principles of Oil Production. McGraw-Hill, New York, 1949.

[53] M. Muskat, R. D. Wyckoff, H. G. Botset, and M. W. Meres. Flow of gas-liquid mixtures through sands. Petrol. Trans. AIME, 123:69-96, 1937.

[54] M. J. Oak. Three-phase relative permeability of water-wet Berea. In SPE/DOE Seventh Symposium on Enhanced Oil Recovery, Tulsa, OK, April 22-25, 1990. (SPE/DOE 20183).

[55] M. J. Oak. Three-phase relative permeability of intermediate-wet Berea sandstone. In SPE Annual Technical Conference and Exhibition, Dallas, TX, October 6-9, 1991. (SPE 22599). 
R. Juanes and T. W. Patzek: Strictly hyperbolic models of three-phase flow

[56] M. J. Oak, L. E. Baker, and D. C. Thomas. Three-phase relative permeability of Berea sandstone. JPT, 42(8):1054-1061, August 1990.

[57] J. S. Osoba, J. G. Richardson, J. K. Kerver, J. A. Hafford, and P. M. Blair. Laboratory measurements of relative permeability. Petrol. Trans. AIME, 192:47-56, 1951.

[58] J. C. Parker and R. J. Lenhard. A model for hysteretic constitutive relations governing multiphase flow, 1. Saturation-pressure relations. Water Resour. Res., 23(12):2187-2196, 1987.

[59] D. W. Peaceman. Fundamentals of Numerical Reservoir Simulation, volume 6 of Developments in Petroleum Science. Elsevier, Amsterdam, 1977.

[60] J. G. Richardson, J. K. Kerver, J. A. Hafford, and J. S. Osoba. Laboratory determination of relative permeability. Petrol. Trans. AIME, 195:187-196, 1952.

[61] A. Sahni, R. Guzman, and M. Blunt. Theoretical analysis of three phase flow experiments in porous media. In SPE r1st Annual Technical Conference and Exhibition, Denver, CO, October 6-9, 1996. (SPE 36664).

[62] D. N. Saraf, J. P. Batycky, C. H. Jackson, and D. B. Fisher. An experimental investigation of three-phase flow of water-oil-gas mixtures through water-wet sandstones. In SPE California Regional Meeting, San Francisco, CA, March 24-26, 1982. (SPE 10761).

[63] D. G. Schaeffer and M. Shearer. The classification of $2 \times 2$ systems of nonstrictly hyperbolic conservation laws, with application to oil recovery. Comm. Pure Appl. Math., 40(1):141-178, 1987. Appendix with D. Marchesin and P. J. Paes-Leme.

[64] F. N. Schneider and W. W. Owens. Sandstone and carbonate two- and three-phase relative permeability characteristics. SPEJ, 10(1):75-84, March 1970. Petrol. Trans. AIME, 249.

[65] M. Shearer. Loss of strict hyperbolicity of the Buckley-Leverett equations for three phase flow in a porous medium. In M. F. Wheeler, editor, Numerical Simulation in Oil Recovery, volume 11 of The IMA Volumes 
R. Juanes and T. W. Patzek: Strictly hyperbolic models of three-phase flow

in Mathematics and its Applications, pages 263-283. Springer-Verlag, New York, 1988.

[66] M. Shearer and J. A. Trangenstein. Loss of real characteristics for models of three-phase flow in a porous medium. Transp. Porous Media, 4:499525, 1989.

[67] J. Smoller. Shock Waves and Reaction-Diffusion Equations, volume 258 of A Series of Comprehensive Studies in Mathematics. Springer-Verlag, New York, second edition, 1994.

[68] H. L. Stone. Probability model for estimating three-phase relative permeability. JPT, 23(2):214-218, February 1970. Petrol. Trans. AIME, 249.

[69] H. L. Stone. Estimation of three-phase relative permeability and residual oil data. J. Can. Petrol. Technol., 12(4):53-61, 1973.

[70] J. A. Trangenstein. Three-phase flow with gravity. In W. B. Lindquist, editor, Current Progress in Hyperbolic Systems: Riemann Problems and Computations, volume 100 of Contemporary Mathematics, pages 147159. American Mathematical Society, Providence, RI, 1989.

[71] C. Truesdell and W. Noll. The Non-Linear Field Theories of Mechanics. Springer-Verlag, Berlin, 1965. (Second edition, 1992).

[72] M. I. van Dijke, K. S. Sorbie, M. Sohrabi, D. H. Tehrani, and A. Danesh. Three-phase flow in WAG processes in mixed-wet porous media: porescale network simulations and comparison with micromodel experiments. In SPE/DOE Thirteenth Symposium on Improved Oil Recovery, Tulsa, OK, 2002. (SPE 75192).

[73] H. J. Welge. A simplified method for computing oil recovery by gas or water drive. Petrol. Trans. AIME, 195:91-98, 1952.

[74] R. D. Wyckoff and H. G. Botset. The flow of gas-liquid mixtures through unconsolidated sands. Physics, 7:325-345, 1936.

[75] E. Zauderer. Partial Differential Equations of Applied Mathematics. Series in Pure and Applied Mathematics. John Wiley \& Sons, New York, 1983. 